\title{
A Machine Learning Method with Threshold Based Parallel Feature Fusion and Feature Selection for Automated Gait Recognition
}

\author{
Muhammad Sharif, Department of CS, COMSATS University Islamabad, Wah Campus, Wah Cantt, Pakistan \\ Muhammad Attique, Department of Computer Science, HITEC University, Museum Road Taxila, Pakistan \\ Muhammad Zeeshan Tahir, Department of CS, COMSATS University Islamabad, Wah Campus, Wah Cantt, Pakistan \\ Mussarat Yasmim, Department of CS, COMSATS University Islamabad, Wah Campus, Wah Cantt, Pakistan \\ Tanzila Saba, Artificial Intelligence \& Data Analytics (AIDA) Lab, CCIS Prince Sultan University, Riyadh, Saudi Arabia \\ Urcun John Tanik, Texas A\&M University-Commerce, Commerce, USA
}

\begin{abstract}
Gait is a vital biometric process for human identification in the domain of machine learning. In this article, a new method is implemented for human gait recognition based on accurate segmentation and multi-level features extraction. Four major steps are performed including: a) enhancement of motion region in frame by the implementation of linear transformation with HSI color space; $b$ ) Region of Interest (ROI) detection based on parallel implementation of optical flow and background subtraction; c) shape and geometric features extraction and parallel fusion; d) Multi-class support vector machine (MSVM) utilization for recognition. The presented approach reduces error rate and increases the CCR. Extensive experiments are done on three data sets namely CASIA-A, CASIA-B and CASIA-C which present different variations in clothing and carrying conditions. The proposed method achieved maximum recognition results of $98.6 \%$ on CASIA-A, $93.5 \%$ on CASIA-B and 97.3\% on CASIA-C, respectively.
\end{abstract}

\section{KEYWORDS}

Gait Recognition, Geometric Features, HOG Features, MSVM Classifier, Segmentation

\section{INTRODUCTION}

In the domain of computer vision, Human Gait Recognition (HGR) is an active research area due to its unobtrusiveness for identification and verification. In comparison with other biometric devices such as iris and face detection (Choudhury and Tjahjadi, 2015; Lishani, Boubchir et al., 2017; Dey, Ashour et al., 2018; Wang, Li et al., 2018), the gait offers an opportunity to recognize human even at a distance point by utilizing a video camera. Existing research in this area has explained that gait is likely to become a robust biometric for assistance in many applications such as surveillance in airports, bus stations, clinical analysis, banks surveillance systems and forensic applications (Aqmar, Fujihara et al., 2014; Yasmin, Sharif et al., 2016; Zeng and Wang, 2016).

Recently, several image processing and machine learning-based techniques are introduced for HGR. These techniques can be separated into two classes such as model based (MB) (Bashir, Xiang, \& Gong, 2010) and model free (MF) (Zeng, Wang, \& Li, 2014). The MB approach takes structural 
model of a human body in the absence of motion. Afterwards, parameters of these structural models are further applied as features like joint angles. The major advantage of this approach is to express high-level model of a human body but it is complex because of high cost and computational time. This approach also works better over the view invariant and cofactors like carrying, clothing and shadows which affects the recognition rate (Zeng, Wang et al., 2014, Arora, Hanmandlu et al., 2015). The model free class operates on silhouette images of a human body instead of a structural model. This approach is less sensitive for the quality of an image as compared to model-based class. Also, it has low computational time and cost. The major challenge of this approach is sensitivity because of several problems such as carrying, clothing and shadows (Piccardi 2004, Rida, Jiang et al., 2016; Wu, Huang et al., 2017).

A general gait recognition framework consists of several sub steps such as preprocessing, ROI detection, feature extraction and recognition. The preprocessing step has much importance for obtaining high recognition rate because the raw input videos have a complex background and low contrast. The low contrast videos affect ROI detection which later on degrades the recognition accuracy. Therefore, preprocessing is a major step for improving the contrast of input video and also removes extra noise such as change in background, variations and human change. In gait recognition, different segmentation methods have been introduced for ROI detection such as background subtraction, thresholding, watershed and few more (Piccardi, 2004; Gupta, Dixit et al., 2014). There are several types of features which exist in computer vision such as texture, color, geometric, shape, Gabor and wavelet transform. These features are high in number of dimensions; therefore, they reduce the accuracy. This kind of problem is resolved by several researchers by the implementation of reduction techniques. The major reduction techniques are Principal Component Analysis (PCA) (Ryu and Kamata, 2011), ICA, genetic algorithm and few more (Khan, Sharif et al., 2016). Finally, the reduced features are utilized by supervised learning methods as SVM, Fine K-Nearest Neighbor (FKNN), neural network, decision trees and regression models (Abdullah and El-Alfy, 2015; Khan, Sharif et al., 2016; Nida, Sharif et al., 2016). These methods perform significantly well when the extracted number of features is unique and have no redundancy between them. Therefore, classification accuracy fully depends on the extracted number of features.

In literature, numerous researchers introduced several methods for HGR. However, the performance of HGR is significantly affected due to several covariate aspects such as change in clothes, occlusion, view angle, time, carrying conditions and different variations (Al-Tayyan, Assaleh et al., 2017, Yu, Chen et al., 2017). In this article, several challenges are taken into account such as the presence of shadows, varying view point at different conditions, effects of occlusion, change in human angle, change in viewpoint and high dimension of extracted features. A new method is presented in this work which resolves the listed problems for HGR. The proposed method is based on three pipe line procedures: a) a preprocessing method is implemented for distinguishing the human from background, which is a combination of HSI color transformation and linear contrast enhancement; b) implementation of a uniform segmentation method and optimizing results by fusion of background subtraction; c) fused texture and shape features based on parallel method and selecting the best features using minimum distance values. The selected features are fed to Multi category AVM (MSVM) for recognition.

The contributions of this article are listed below:

1) In preprocessing, a hybrid technique is implemented for contrast enhancement based on HSI color transformation. The Hue channel is selected, and foreground contrast is increased by using a logarithmic function. Finally, the logarithmic image is optimized by performing linear contrast which efficiently distinguished background and foreground objects.

2) A uniform segmentation method is implemented for ROI detection which improves the segmentation performance by fusion of background subtraction. The fusion process is done by mutual information method. 
3) The texture, geometric and shape features are fused based on the parallel method and best features are selected by minimum distance value features. The minimum distance features are selected by their threshold value.

\section{BACKGROUND}

In literature, many image processing and machine learning-based techniques have been implemented for automated HGR. For HGR, features extraction is much important for achieving highest accuracy. Recently, features extraction techniques are mostly utilized for several computer vision applications (Fernandes and Bala, 2016; Amin, Sharif et al., 2017; Amin, Sharif et al., 2017; Fernandes, Gurupur et al., 2017; Rajinikanth, Madhavaraja et al., 2017; Rajinikanth, Satapathy et al., 2017; Shah, Chen et al., 2017; Shah, Sharif et al., 2017; Amin, Sharif et al., 2018; Ansari, Shah et al., 2018; Bhushan, Danti et al., 2018; Bokhari, Syedia et al., 2018; Naqi, Sharif et al., 2018; Raja, Fernandes et al., 2018; Rajinikanth, Fernandes et al., 2018; Ranjan, Arya et al., 2018). The researchers had shown close attention for the view invariant HGR. Lishani et al. (Lishani, Boubchir et al., 2014) introduced a supervised features extraction method that is useful for choosing the ideal features for HGR. The introduced method efficiently works under several effects and variations such as wearing and carrying conditions. The Harlick features are extracted from gait energy image and show improved performance on CASIA gait data set. Lishani et al. (Lishani, Boubchir et al., 2016) focused on supervised features extraction method to select the best features under several challenges and conditions such as wearing, carrying and few more. The Gabor filter-based features are extracted and show significant recognition performance under the variation effects. Zeng at al. (Zeng, Wang et al., 2014) presented a MB approach for HGR based on Deterministic Learning (DL). The silhouette based lower limb joint angles are utilized for features extraction which is later identified the by Radial Basis Function (RBF) neural network and DL method. The CASIA-A and CASIA-B data sets are utilized for evaluation having recognition rate $92.5 \%$ and $91.9 \%$, respectively. Wang et al. (Wang, Zhang et al., 2012) introduced a new temporal template method called Chrono Gait Image (CGI) for explaining the spatial-temporal walking to identify human gait and to improve recognition performance. The contour images are extracted from their corresponding silhouette images by utilizing local entropy method and their temporal information is added into CGI by utilizing the multi-channel method. Experiments are conducted on three data sets and show improved performance. Liu et al. (Liu, Yin et al., 2011) introduced a new method for HGR by extraction gait features of outer most contour in the frame. The extracted features are high in dimension which can be reduced by using PCA. Afterwards, Multiple Discriminant Analysis (MDA) method is utilized for the optimization of reduced features and Nearest Neighbor and Exemplars Nearest Neighbor (ENN) classifiers are utilized for producing final feature vector for classification by Multi-dimensional Scaling (MDS). The evaluation results show improved performance of the introduced method. Hu et al. (Hu, Wang et al., 2013) presented a new framework for HGR. The presented scheme is built on silhouette images which are replaced with Local Binary Pattern (LBP) flow. This flow works better for color images and gives lot of details with the help of shape information and shows effectiveness in gait recognition. This method also reduces the computational time. Zeng et al. (Zeng and Wang, 2013) described a HGR approach based on silhouette features. It combines spatiotemporal motion features and physical parameters of human body to analyze shape parameters. The method is just verified for the sequence with a lateral view. Rida et al. (Rida, Jiang et al., 2016) described a new framework for the selection of most discriminative parts of human body. This method reduces intra class variation and increases recognition accuracy using CASIA data set. Rida et al. (Rida, Almaadeed et al., 2016) discussed a new method based on supervised feature extraction that is capable to choose important discriminative features of human body in the absence of covariate factors using CASIA gait database. Lishani et al. (Lishani, Boubchir et al., 2017) extracted detailed wavelet features for HGR. The features are calculated in the dynamic area of Gait Energy Image (GEI) using Haar wavelet decomposition. 
The extracted features are reduced by Spectral Regression Kernel Discriminant Analysis (SRKDA) method and ideal features are selected for classification. The KNN classifier is used for classification and evaluation is performed on CASIA gait data set with recognition rate $93 \%$. Munif et al. (Alotaibi and Mahmood, 2015) introduced a Convolutional Neural Network (CNN) model for HGR which is less sensitive to several types of challenges such as variation and occlusion. The major advantage of introduced method is that it can be easily implemented on small data sets without any fine tuning and augmentation. The introduced method performs significantly well on CASIA B data set and achieved $86.50 \%$ accuracy on different cross view sequences. Islam et al. (Islam, Islam et al., 2017) introduced a new HGR method using wavelet-based features. The frequency domain description of input sequence is achieved by Fast Fourier Transform (FFT). Later on, a spectrum is obtained by Discrete Wavelet Transform (DWT) and four coefficients are calculated by single level wavelet. Finally, entropy-based features are calculated from these wavelets and classified by template matching. The results reveal outperformance as compared to existing methods. A summary of gait recognition techniques in literature is presented in Table 1.

\section{PROPOSED METHOD}

The proposed HGR approach comprises of four steps as shown in Figure 1. The preprocessing step improves human region in the given video sequence and later on extracts human region by implementing uniform segmentation and optimizing its effects by fusion of background subtraction. Texture, geometric and shape features are extracted from ROI and parallel fusion is performed. Afterwards, a features selection algorithm is implemented to select the best features from fused vector based on minimum distance that are later recognized by One against all SVM.

Table 1. Summary of existing gait recognition techniques

\begin{tabular}{|c|c|c|c|c|}
\hline Reference & Technique & Features & Classification & Dataset \\
\hline $\begin{array}{l}\text { (Lishani, Boubchir et } \\
\text { al., 2014) }\end{array}$ & $\begin{array}{l}\text { Harlick descriptors } \\
\text { with SVM approach } \\
\text { for GEI based } \\
\text { recognition }\end{array}$ & Harlick & One against all SVM & CASIA-B \\
\hline $\begin{array}{l}\text { (Lishani, Boubchir et } \\
\text { al., 2016) }\end{array}$ & $\begin{array}{l}\text { Gabor features with } \\
\text { SRKDA }\end{array}$ & Gabor & SRKDA & CASIA-B \\
\hline $\begin{array}{l}\text { (Zeng, Wang et al., } \\
\text { 2014) }\end{array}$ & DL based recognition & Lowe limb patterns & RBF neural Network & $\begin{array}{l}\text { CASIA-A and } \\
\text { CASIA-B }\end{array}$ \\
\hline $\begin{array}{l}\text { (Wang, Zhang et al., } \\
\text { 2012) }\end{array}$ & CGI approach & Entropy features & $\begin{array}{l}\text { 1-Nearest Neighbor } \\
(1-\mathrm{NN})\end{array}$ & $\begin{array}{l}\text { USF HumanID and } \\
\text { CASIA-B }\end{array}$ \\
\hline (Liu, Yin et al., 2011) & $\begin{array}{l}\text { Outermost contour } \\
\text { approach }\end{array}$ & $\begin{array}{l}\text { Outermost contour } \\
\text { features }\end{array}$ & KNN and ENN & CASIA-B \\
\hline $\begin{array}{l}\text { (Hu, Wang et al., } \\
\text { 2013) }\end{array}$ & $\begin{array}{l}\text { Incremental learning } \\
\text { with LBP flow } \\
\text { approach }\end{array}$ & LBP features & HMM & $\begin{array}{l}\text { CASIA-A and } \\
\text { CASIA-B }\end{array}$ \\
\hline $\begin{array}{l}\text { (Zeng and Wang, } \\
\text { 2013) }\end{array}$ & $\begin{array}{l}\text { DL based gait } \\
\text { recognition }\end{array}$ & $\begin{array}{l}\text { Spatiotemporal } \\
\text { motion and physical } \\
\text { features }\end{array}$ & RBF NN & $\begin{array}{l}\text { CASIA, CMU MoBo } \\
\text { and TUM GAID }\end{array}$ \\
\hline $\begin{array}{l}\text { (Alotaibi and } \\
\text { Mahmood, 2015) }\end{array}$ & CNN based approach & Deep features & $\mathrm{CNN}$ & CASIA-B \\
\hline $\begin{array}{l}\text { (Islam, Islam et al., } \\
\text { 2017) }\end{array}$ & $\begin{array}{l}\text { Entropy based } \\
\text { features approach }\end{array}$ & Wavelet features & Template matching & CASIA-B \\
\hline
\end{tabular}




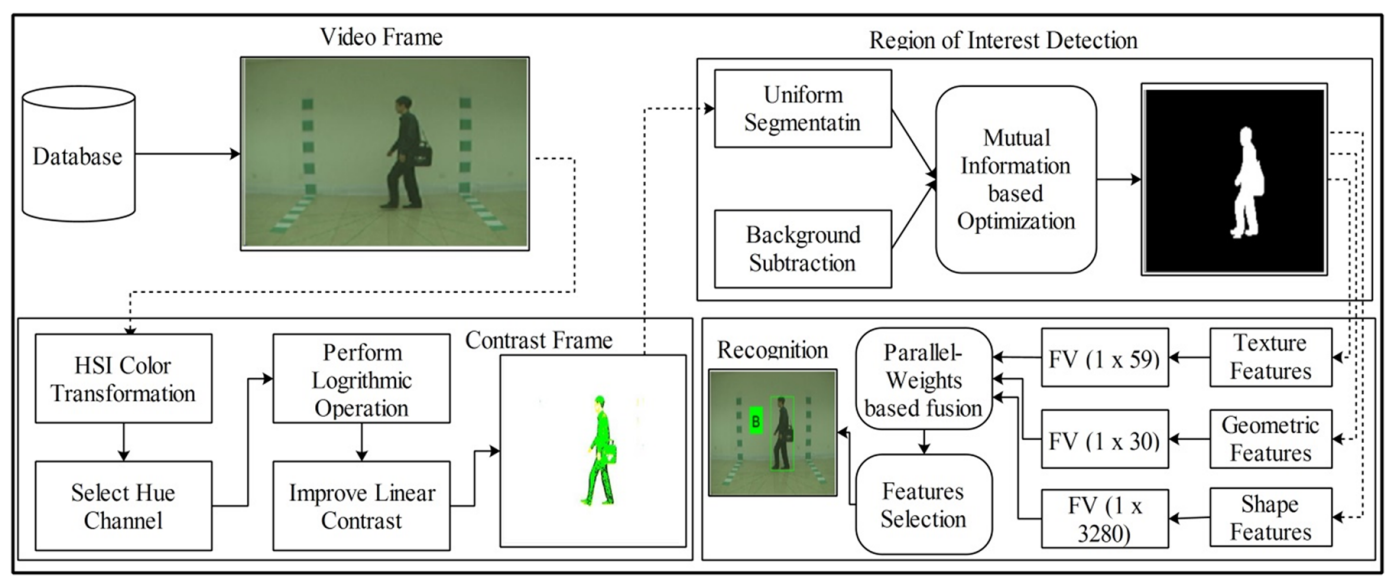

\section{Preprocessing}

In the field of computer vision, preprocessing step plays a key part in improving the quality of input image (Raja, Fernandes et al., 2018). In video sequences, preprocessing step improves the subjective quality of extracted frames. Low contrast and variation in extracted frames create problems for human extraction; hence preprocessing step is used to resolve the problem of occlusion and motion analysis in given video sequences. This step also helps in tracing relevant features for the segmentation step. In this article, a new hybrid preprocessing approach is designed in which initially HSI color transformation is extracted to differentiate between human and background. Afterwards, hue channel is selected based on good visual results and a weighted logarithmic function is performed for improving the brightness of moving parts. Finally, a linear contrast function is applied to a weighted logarithmic frame for obtaining a new enhanced frame which possesses significantly good visual quality as compared to the original extracted frame. The detailed description is given below.

Let $\Phi$ denotes the given video sequences having several extracted frames denoted by $3 / 4(x, y)$. The original video frame $3 / 4(x, y)$ is a combination of red, green and blue channels which are extracted as follows in Equation (1):

$$
\left(\begin{array}{l}
3 / 4=\frac{r}{3 / 4(x, y)} \\
3 / 4_{\mathrm{G}}=\frac{\mathrm{g}}{3 / 4(\mathrm{x}, \mathrm{y})} \\
3 / \mathrm{B}_{\mathrm{B}}=\frac{\mathrm{r}}{3 / 4(\mathrm{x}, \mathrm{y})}
\end{array}\right)
$$

Where, $3 / 4,3 / 4,3 / 4$ denote the extracted red, green and blue channels, respectively. In preprocessing, initially HIS color transformation is performed on original frame and Hue channel is extracted from it based on high intensity value and more information as compared to $\mathrm{S}$ and I channels. The hue channel is extracted by Equation (2) and Equation (3) below: 
$3 /{ }_{\mathrm{H}}(\mathrm{x}, \mathrm{y})= \begin{cases}\theta & \text { if } 3 / \mathrm{B}_{\mathrm{B}} \leq 3 / \mathrm{A}_{\mathrm{G}} \\ 360^{\circ}-\theta & \text { if } 3 / \mathrm{B}_{\mathrm{B}}>3 / \mathrm{A}_{\mathrm{G}}\end{cases}$

$=\cos ^{-1}\left\{\frac{\frac{1}{2}\left[\left(3 / \mathrm{R}_{\mathrm{R}}-3 / \mathrm{G}_{\mathrm{G}}\right)+\left(3 / \mathrm{A}_{\mathrm{R}}-3 / \mathrm{B}_{\mathrm{B}}\right)\right]}{\sqrt{\left[\left(3 / \mathrm{A}_{\mathrm{R}}-3 / \mathrm{G}_{\mathrm{G}}\right)^{2}+\left(3 / \mathrm{A}_{\mathrm{R}}-3 / \mathrm{B}_{\mathrm{B}}\right)\left(3 / \mathrm{A}_{\mathrm{G}}-3 / \mathrm{B}_{\mathrm{B}}\right)\right]}}\right.$

The effects of HSI color transformation are shown in Figure 2(b) and selection of hue channel is shown in Figure 2(c).

After that, a weighted logarithmic function is implemented which controls the variation and human movement in given video sequences. The major purpose of this weighted function is to highlight moving area in the video. The weighted logarithmic function is defined as in Equation (4) and Equation (5):

$3 / 4(x, y)=\dot{E} \times \log (a+3 / 4(x, y))$

$\mathrm{E}(\mathrm{i})=\sum_{\mathrm{i}=0}^{1} 3 / \mathrm{H}_{\mathrm{H}}(\mathrm{i})$

Where, É $(i)$ denotes the weight value which is between $\{0,1\}$. The weight value of each frame is calculated by their pixels values. The parameter a denotes a constant value initialized as 10 . The weighted logarithmic function effects are shown in Figure 2(d). Finally, a piece wise linear contrast method is performed, and a new enhanced frame is obtained which efficiently separates human from the background as shown in Figure 2(e). The linear contrast is defined as follows:

Let the starting point of $3 / 4(\mathrm{x}, \mathrm{y})$ luminance is $\mathrm{x}_{1}, \mathrm{l}=0,1,2,3, \ldots 1-1$ and the starting position of output luminance is $\mathrm{y}_{1}, \mathrm{l}=0,1,2,3, \ldots l-1$. Then the $1-1$ transform function is defined as follows in Equation (6):

$3 / 4-1(x, y)=\frac{y_{1}-y_{1-1}}{x_{1}-x_{1-1}} \times\left(x-x_{1-1}\right)+y_{1-1}$

Where, ${ }^{3 / 4}(x, y)$ is final enhanced frame as shown in Figure $2(e)$. The enhanced frame is further utilized in the next section for human extraction through optical flow and background subtraction.

\section{Human Extraction}

In this section, human is extracted from frames using image processing methods such as background subtraction and optical flow. The human extraction is an important step for identifying the gait such as carrying bag, normal walk etc. The basic goal of human extraction is to detect ROI in the given sequence. The ROI is used to extract useful gait features which improve the recognition accuracy. In 
Figure 2. Frame preprocessing: a) original frame; b) HSI color transformation; c) Selection of hue channel; d) Weighted log transformation; e) Linear contrast stretching

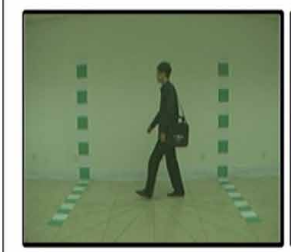

(a)

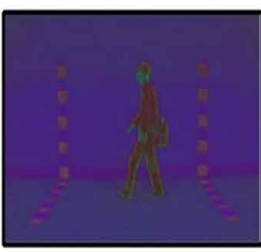

(b)

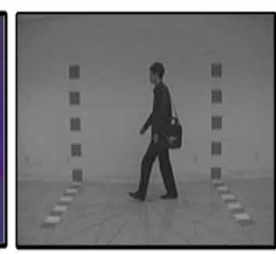

(c)

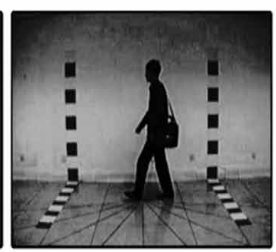

(d)

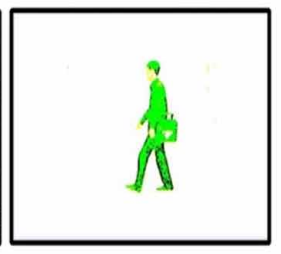

(e)

this article, initially the motion features are extracted using optical flow for which Horn-Schunck method (Barron 1992) is utilized. The enhanced $3 / 4_{-1}(x, y)$ frame is set as an input to Horn-Schunck method which gives the most prominent motion features in terms of speed and direction of human movement in frame $3 /{ }_{1-1}(x, y)$. It estimates the motion of foreground in time $t$ to $t+1$ and provides the information of moving regions in three directions such as horizontal, vertical and time. The frame contrast at time $\mathrm{t}$ with spatial points $(\mathrm{u}, \mathrm{v})$ is defined by * $(\mathrm{u}, \mathrm{v}, \mathrm{t})$ in Equation (7).

$\cdot(\mathrm{u}, \mathrm{v}, \mathrm{t})=\cdot(\mathrm{u}+\Delta \mathrm{x}, \mathrm{v}+\Delta \mathrm{y}, \mathrm{t}+\Delta \mathrm{t})$

Then the contrast constancy assumption relation leads to the equation with chain rule as given in Equation (8) below:

$\cdot{ }_{\mathrm{u}} \mathrm{x}+{ }_{\mathrm{v}} \mathrm{y}+{ }_{\mathrm{t}}=0$

Where, $\mathrm{x}, \mathrm{y}$ are the parallel and vertical vectors of optical flow, respectively. Then the value of change of foreground contrast with the constraint of smoothness is set to minimize using the sum of errors shown in Equation (9):

$\left(\cdot_{\mathrm{u}^{\mathrm{g}}}+{ } \mathrm{v}_{\mathrm{v}^{y}}+{ }_{\mathrm{t}}\right)^{2}+¥\left(\mathrm{~g}_{\mathrm{u}^{2}}+\mathrm{g}_{\mathrm{v}^{2}}+\propto_{\mathrm{u}^{2}}-\propto_{\mathrm{v}^{2}}\right) \mathrm{dudv}$

After that, the moving region is segmented by background subtraction (Premalatha, Williams et al., 2016). The Sobel filter is used to draw edges on motion frame to localize the fragment object. The Sobel filter is defined as in Equation (10):

$\mathrm{E}=\tilde{\mathrm{A}}(\cdot \cdot)$, where $\tilde{\mathrm{A}} \in$ Sobel filter

After calculation of human edges, a $5 \times 5$ matrix is constructed as in Equation (11) which contains foreground and background pixels. 


$$
M=\left[\begin{array}{lllll}
0 & 0 & 0 & 0 & 0 \\
0 & 1 & 1 & 1 & 0 \\
0 & 1 & 1 & 1 & 0 \\
0 & 1 & 1 & 1 & 0 \\
0 & 0 & 0 & 0 & 0
\end{array}\right]
$$

In the above equation, 1's denote the foreground pixels and 0's as background. A convolution operation is implemented to combine edges of foreground and background pixels. Equation (12) defines the convolution function.

smoothing $=\operatorname{con} 2(\mathrm{E}, \mathrm{M})$

The convolution filtering is used to modify the spatial properties of given frame because convolution is a general-purpose filter which affects given frame or image. An integer matrix of size $5 \times 5$ is applied on motion frame to determine the value of central pixel by the sum of all neighbors of weighted values. Finally, the mean value of resultant image is calculated which consists of foreground and background. The mean value is utilized to set a threshold function for final binary image as follows in Equation (13):

$\operatorname{Threshold}(\mathrm{x}, \mathrm{y})= \begin{cases}0 & \text { if }\left|\mathrm{F}_{\mathrm{i}+1}-\mathrm{F}_{\mathrm{i}}\right|<\mu \\ 1 & \text { if }\left|\mathrm{F}_{\mathrm{i}+1}-\mathrm{F}_{\mathrm{i}}\right| \geq 1 / 4\end{cases}$

The above equation shows that if difference between $F_{i+1}$ and $F_{i}$ is less than mean value, background is considered otherwise foreground. The effects of human extraction using optical flow and improved background subtraction are shown in Figure 3 and Figure 4.

\section{Features Extraction}

Features extraction plays a key role for the representation of an image in machine learning domain. In gait recognition, the raw video sequences are very large; therefore, very high computational support is required. Moreover, several hidden characteristics are involved in the raw frames which are not visible easily. In addition, there exists much irrelevant and redundant information which affects the system accuracy. Therefore, strong and efficient feature set is required for any recognition system. In this article, Histogram of Oriented Gradients (HOG), geometric and Local Binary Pattern (LBP) features are calculated for the construction of a code book by parallel fusion. The extracted features consist of texture, geometric and shape. All features are extracted from ROI. The detailed description of each extracted feature set is given below and also an overview is given in Figure 5.

\section{Shape Features}

In this section, HOG features (Khan, Sharif et al., 2017) are extracted known as shape features. The HOG features are easy to express the shape of an object and are robust to variations in geometry and illumination changes. The ROI frame having size $128 \times 64$ is used for HOG features extraction. The major steps in computation of HOG features are: a) compute the gradients; b) orientation binning such as cells and votes; c) descriptor blocks, and d) block normalization. After these steps, a vector is generated of size $1 \times 3780$ which is reduced by PCA. The major reason of features reduction is that the high dimensional features degrade the system accuracy. Therefore, through PCA, a reduced vector is obtained of size $1 \times 1000$. 
Figure 3. ROI extraction using optical flow and improved background subtraction. a) Original frame; b) Horn-Schunck optical flow; c) Improved background subtraction; d) ROI extraction.

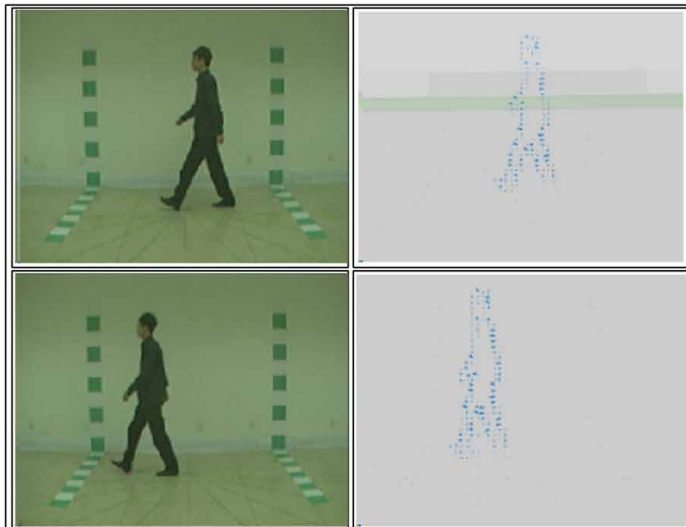

(a)

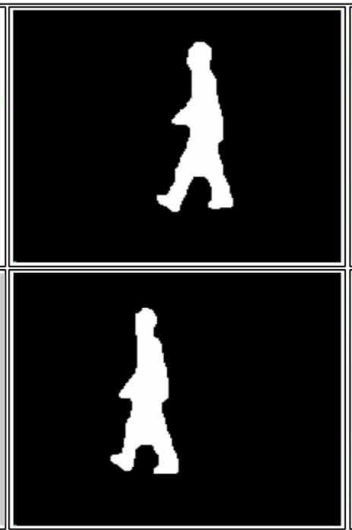

(c)

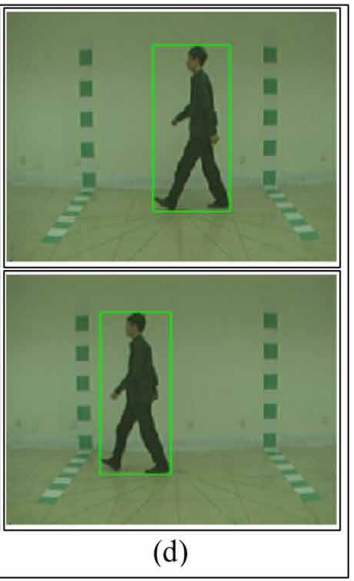

(d)

Figure 4. Human extraction results: a) Original frame; b) HSI transformation; c) Improved background subtraction frame.
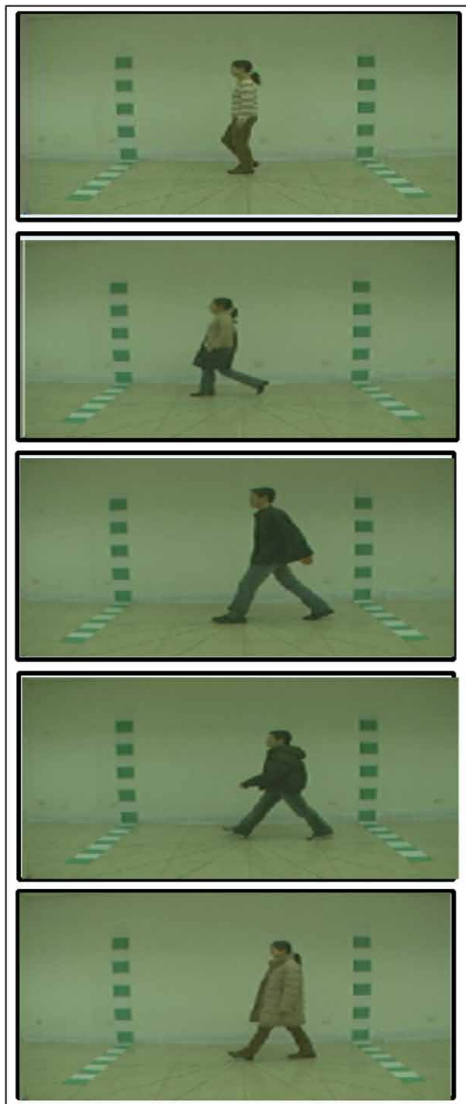

(a)
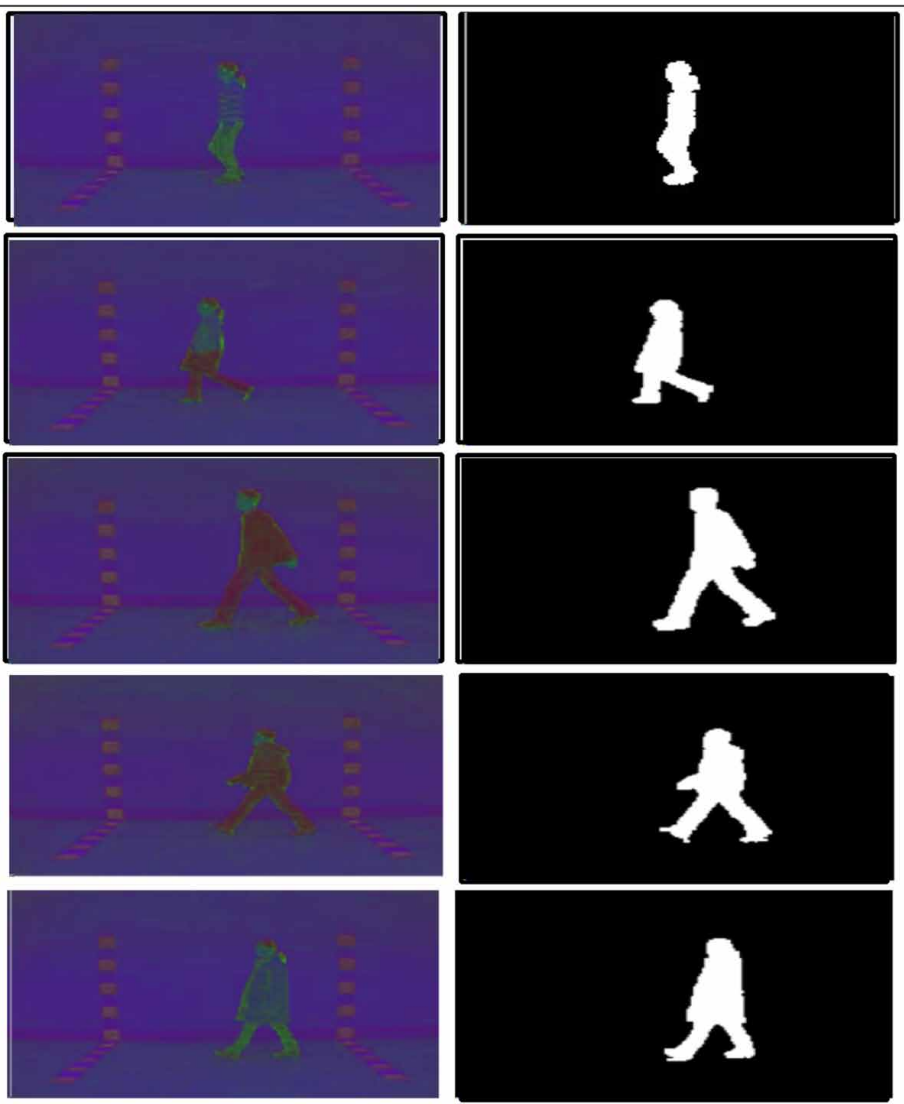

(b) 


\section{Geometric Features}

In the field of machine learning, geometric features are extracted to determine the difficulty of optical tasks in the given scene. The major goal of geometric descriptor is to find a set of representative features. The geometric features significantly solve the recognition problem and also perform well in video sequences to predict the subsequence actions. In this article, six geometric features are calculated as given in Table 2 (Moustakas, Tzovaras et al., 2010). For each geometric feature, five statistical parameters are extracted such as mean, SD, variance, skewness and SVD. After that, all geometric features are combined in one vector.

\section{Texture Features}

In video sequences, the extraction of texture features has much attention because of their temporal extent, spatial extent and challenging background as compared to static background. There exist several challenges for dynamic texture features extraction in given video sequences such as a combination of shape and motion features, extraction of robust features, illumination factor and high variation between foreground and background. In this article, the above listed challenges are resolved by volume local binary pattern (VLBP) (Zhao and Pietikainen 2007). The segmented frame is given as an input for texture features extraction. The simple features extraction function of VLBP is defined as below in Equation (14) and Equation (15):

$\mathrm{VLBP}_{\mathrm{l}, \mathrm{p}, \mathrm{r}}=\sum_{\mathrm{q}=0}^{3 \mathrm{p}+1} \mathrm{~V}_{\mathrm{q}} 2^{\mathrm{q}}$

$\mathrm{VLBP}_{\text {vector }}=2^{3 \mathrm{p}+2}$

Table 2. Description of extracted geometric features

\begin{tabular}{|l|l|}
\hline \multicolumn{1}{|c|}{ Feature Name } & \multicolumn{1}{c|}{ Description } \\
\hline Area & $\varphi^{\mathrm{A}}=\sum_{i=1}^{n} \sum_{j=1}^{m} A[i, j]$ \\
\hline Major Axis Length & $\varphi^{\max }=a_{1}+a_{2}$, where $a_{1}, a_{2}$ are distance points of ellipse \\
\hline Minor Axis Length & $\varphi^{\text {min }}=\sqrt{\left(a_{1}+a_{2}\right)^{2}-d}$ \\
\hline Orientation & Angle between x-axis and major axis of ellipse \\
\hline Perimeter & $\varphi^{\rho}=2 l+2 w$, where 1 describes length and w is width \\
\hline Extent & $\varphi^{\varepsilon}=\frac{\varphi^{A}}{\text { Areaofboundingbox }}$ \\
\hline
\end{tabular}


Where, 1 denotes time interval, $\mathrm{p}$ represents number of local neighboring points around central pixels of given frame, $r$ shows the radius and $q=1-p$. Here, $p$ is initialized as 4 numbers of neighboring pixels. Hence the size of extracted VLBP feature is $1 \times 16384$. The size of extracted vector is very big which affects the system computational time. Therefore, to optimize the extracted VLBP features, entropy is implemented on extracted vector and sort into ascending order. After that, top 1000 higher value features are selected which later on are fused with geometric and shape features. This is shown in Equation (16) and Equation (17) below.

$$
\begin{aligned}
& \operatorname{Entropy}(\text { tex })=-\sum_{\mathrm{i}=1}^{\mathrm{N}_{\text {features }}} \mathrm{p}\left(\operatorname{VLBP}_{\mathrm{i}}\right) \log \mathrm{p}\left(\operatorname{VLBP}_{\mathrm{i}}\right) \\
& \operatorname{Vec}(\mathrm{VLBP})=\operatorname{Entropy}(\text { tex })\left[\operatorname{VLBP}_{\text {vector }},{ }^{2}\right]
\end{aligned}
$$

Where, Entropy (tex) denotes entropy calculation function, p shows probability of VLBP feature vector, Vec(VLBP) is final VLBP selected vector of size $1 \times 1000$ and $\beta$ is sorting parameter.

\section{Features Fusion}

Fusion of multiple information in one vector is one of the emerging technologies in computer vision and machine learning based on their several applications such as action recognition, handwritten text recognition, speech recognition, medical imaging, agriculture imaging and face recognition (Khan, Sharif et al., 2017; Sharif, Khan et al., 2017; Khan, Akram et al., 2018; Khan, Akram et al., 2018; Liaqat, Khan et al., 2018; Nasir, Attique Khan et al., 2018; Raza, Sharif et al., 2018; Sharif, Khan et al., 2018; Sharif, Khan et al., 2018). The major aim of features fusion is to combine the discriminative and strong information of all extracted features in one vector to give better performance and improve the system execution time (Fernandes and Bala 2016). However, this step has a limitation of data redundancy and addition of irrelevant information. Therefore, in this section, a threshold based parallel fusion method is implemented in which the HOG, texture and geometric features are fused in one vector. The detail description of features fusion is described below.

Suppose $c_{1} c_{2}, c_{3}, \ldots \ldots c_{k}$ are known as human gait classes. Let $\mathbb{C}=\left\{O \mid O \in \mathbb{R}^{K_{c}}\right\}$ is simple class. Given $\xi_{x 1}, \xi_{x 2}$, and $\xi_{x 3}$ are three extracted feature vectors, where $\xi_{x 1} \in$ HOG Feature, $\xi_{x 2} \in$ Geometric Feature and $\xi_{x 3} \in$ TextureVector, respectively. As the dimension of each extracted vector is known such as $\xi_{x 1}=1 \times 1000, \xi_{x 2}=1 \times 30$ and $\xi_{x 3}=1 \times 1000$, so a threshold function is set for parrallel fusion as follows in Equation (18):

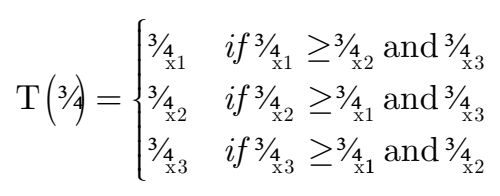

Hence, the size of fused vector is the size of maximum vector as $1 \times 1000$. After that, the most prominent features are selected for final classification. 


\section{Features Selection}

In machine learning applications, features selection techniques show best performance as compared to reduction approaches. In features selection techniques, only best subset of features is chosen whereas in reduction techniques, the original features are changed. The major advantage of features selection techniques is that it consumes minimum time and produces best results, but the limitation is that the prediction level is minimized (Saeys, Inza et al., 2007). In this work, purpose of features selection is to select the best and discriminative features for classification. The idea behind features selection is to reduce the redundancy between features and also improves the system accuracy in terms of correct recognition rate and execution time. For strong features selection, Euclidian distance is calculated between fused features and minimum distance features are selected as shown in Figure 5. Euclidian distance is calculated by Equation (19).

$$
d=\sqrt{\sum_{i=1}^{N}\left(f_{i+1}-f_{i}\right)^{2}}
$$

The minimum distance between two features considers to be the same class and a threshold function is implemented for final selection. The threshold function is defined as follows in Equation (20).

$$
\mathrm{T}(\mathrm{F})= \begin{cases}1 & \text { if } d\left(\mathrm{x}_{\mathrm{i}}, \mathrm{y}_{\mathrm{i}}\right) \in \text { minimum } \\ 0 & \text { if } d\left(\mathrm{x}_{\mathrm{i}}, \mathrm{y}_{\mathrm{i}}\right) \in \text { maximum }\end{cases}
$$

It means that minimum distance features are combined together and maximum distance features are removed from final selected vector. As a result, a new minimum distance vector is obtained which is later fed to MSVM for gait recognition. The cost function of MSVM is defined as: $F_{\mathrm{i}}(\mathrm{x})=\mathrm{C}_{\mathrm{i}}^{\mathrm{T}} \phi(\mathrm{x})+\mathrm{b}_{\mathrm{i}}$ in Equation (21) and Equation (22).

$\operatorname{minimize}: k\left(\tilde{\mathrm{w}}_{\mathrm{i}}, \boldsymbol{q}_{\mathrm{j}}^{\mathrm{i}}\right)=\frac{1}{2}\left\|\tilde{\mathrm{w}}_{\mathrm{i}}\right\|^{2}+\mathrm{D} \sum_{\mathrm{j}=1}^{\tilde{\mathrm{N}}} \mathbf{q}_{\mathrm{j}}^{\mathrm{i}}$

Figure 5. Description of parallel based features fusion and selection

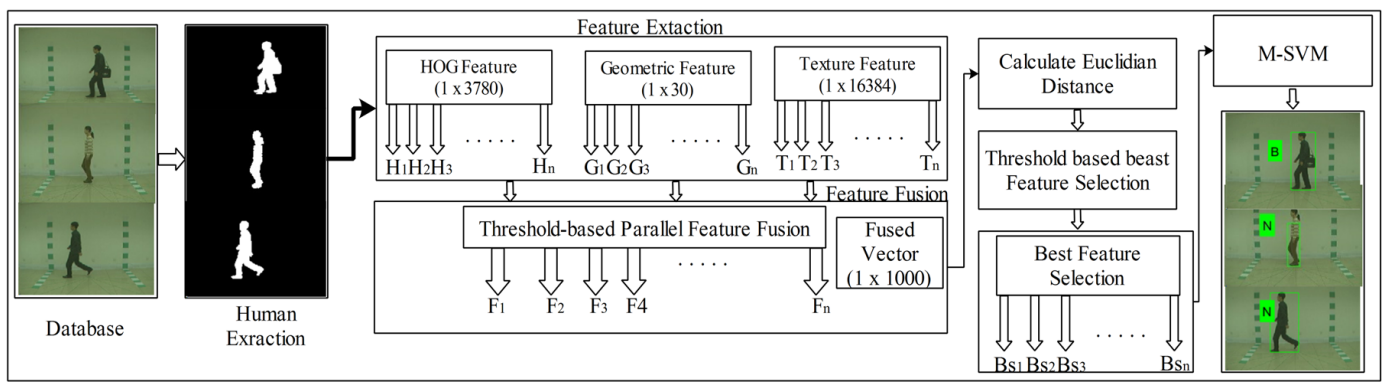


subject to: $\mathrm{y}_{\mathrm{j}}^{\mathrm{C}}\left(\tilde{\mathrm{w}}_{\mathrm{i}}^{\mathrm{T}} \quad \phi\left(\mathrm{x}_{\mathrm{j}}\right)+\mathrm{b}_{\mathrm{i}} \geq 1-\mathbf{q}_{\mathrm{j}}^{\mathrm{i}}, \quad \mathbf{q}_{\mathrm{j}}^{\mathrm{i}} \geq 0\right)$

Where, $\mathrm{y}_{\mathrm{j}}^{\mathrm{c}}=1$ if $\mathrm{y}_{\mathrm{j}}=\mathrm{i}$ else $\mathrm{y}_{\mathrm{j}}=-1$. The proposed recognition effects using video sequences are shown in Figure 6.

\section{Experimental results}

To evaluate the proposed algorithm, three publicly available data sets are selected such as CASIA-A (Wang, Tan et al., 2003), CASIA-B (Wang, Garcia et al., 2017) and CAISA-C (Deng, Wang et al., 2017) as sample frames are shown in Figure 7. Five classification methods such as Decision Tree (DT), LDA, MSVM, FKNN and Ensemble Boosted Tree (EBT) are performed to compare the results of proposed algorithm with standing methods. The performance on selected classification methods are confirmed by seven measures such as True Positive Rate (TPR), False Positive Rate (FPR), Positive Predictive Value (PPV), False Negative Rate (FNR), False Discovery Rate (FDR), Area under the Curve (AUC) (Rajinikanth, Satapathy et al., 2017) and Correct Recognition Rate or Accuracy (CRR). Through CCR, the proposed results are compared with existing approaches. The comparison is done in two steps: a) the recognition results are obtained without features selection and serial based fusion method; $b$ ) the recognition results are obtained by proposed threshold based parallel fusion and features selection method. All results are obtained on MATLAB 2017a having personal computer Core I7 with 16 GB RAM and 8GB graphics card.

\section{CASIA-A Data Set}

The CASIA-A human gait recognition data set consists of 20 subjects fewer than three different views such as lateral, oblique and frontal view in three angles. All videos are captured in an outdoor environment having the frame rate of 25 frames/ sec (FPS). The sample frames are shown in Figure

Figure 6. Visual results of proposed method for human gait recognition with label N (normal walk), W (wearing coat walk) and $B$ (walk with bag)

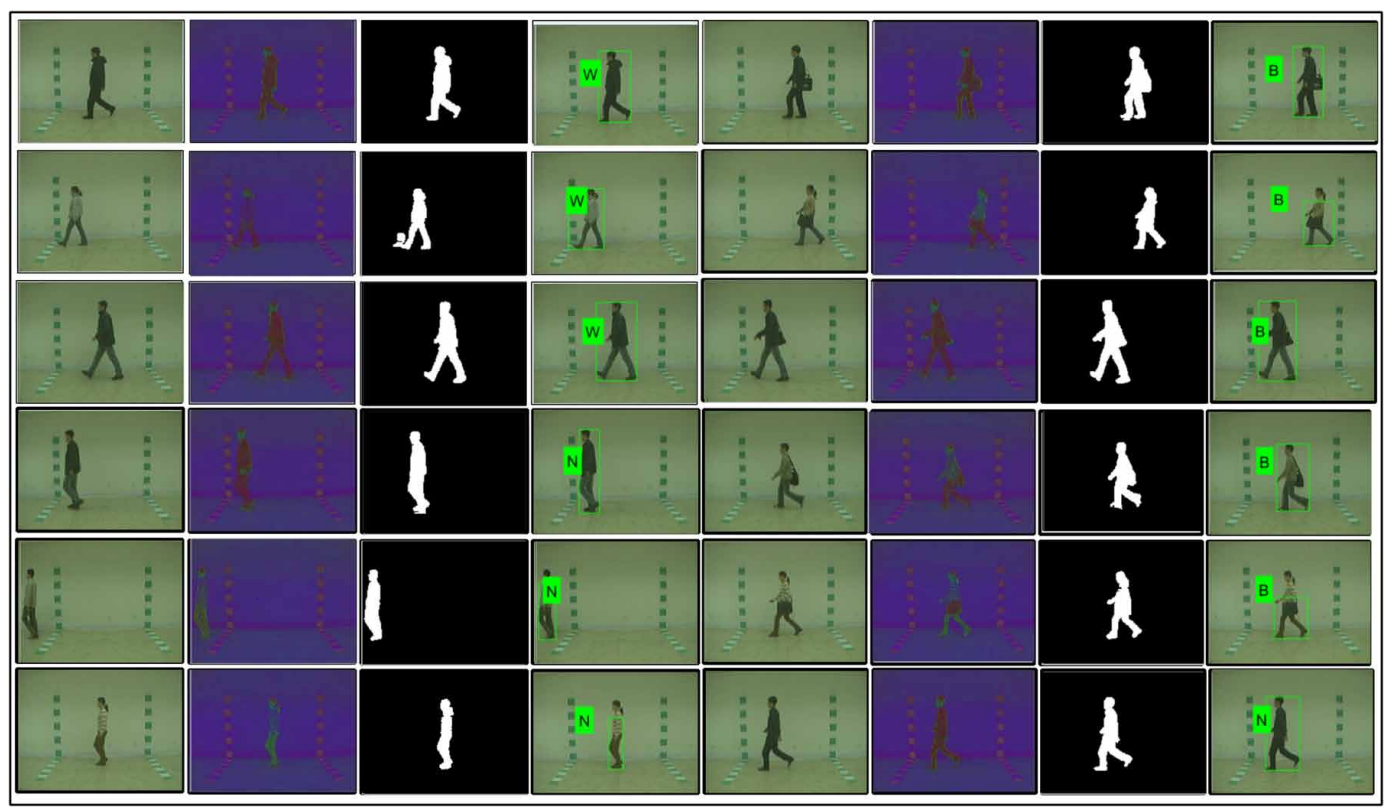




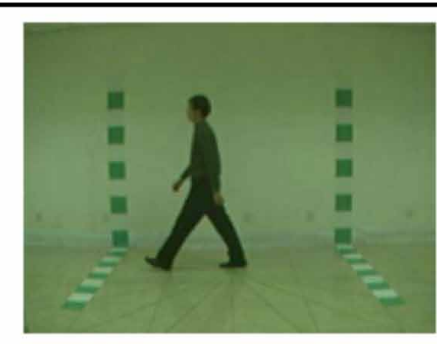

(a) Walking normally

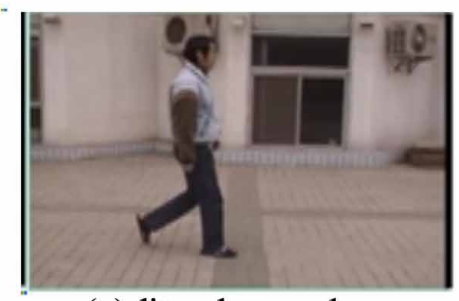

(a) literal zero degree view

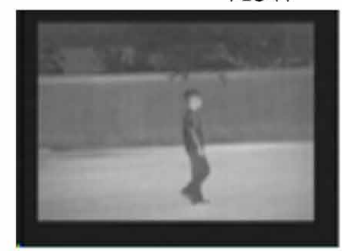

Slow walk

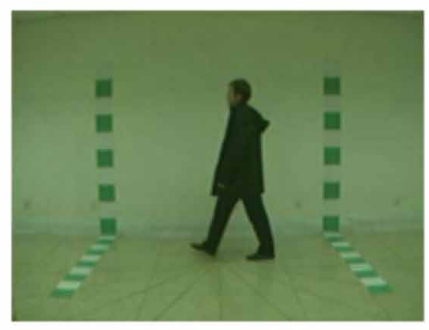

(b) Walking with a coat

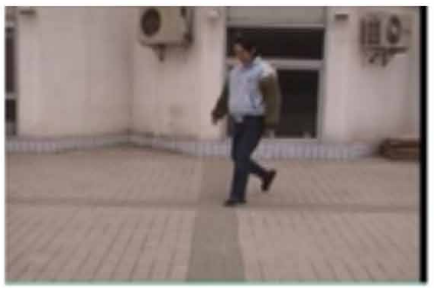

(b) oblique 45 degree view

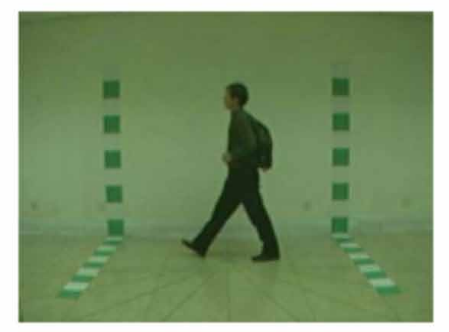

(c) Walking with a bag

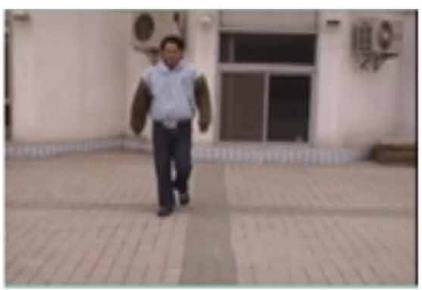

(c) frontal 90 degree view

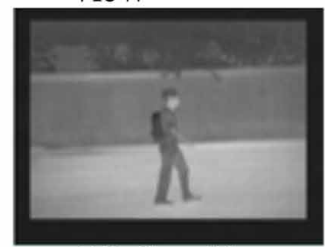

Walk with carrying item

7. A 60:40 approach is adopted for training and testing the system and performs 10-fold validations. The recognition results are evaluated in two steps. First, the recognition results are obtained on serial-based fusion and without feature selection method instead of threshold based parallel fusion and features selection method. The maximum recognition results of this step are achieved on MSVM having recognition rate $98.6 \%$ as given in Table 3. The MSVM accuracy is also proved by Table 4. The recognition results of this step are good, but it consumes too much time for execution. The average execution time of this step is 7.234 FPS. In the second step, proposed system is tested on presented methodology and achieved maximum accuracy $98.9 \%$ as presented in Table 5 and proved by Table 6 . The average execution time of proposed method is 2.301 FPS which is significantly well as compared to step 1 experiment. Moreover, the proposed method is compared with the existing techniques as presented in Table 7 which shows the authenticity of presented method.

\section{CASIA-B Data Set}

This dataset consists of total 124 subjects including 93 male subjects and 31 females. It is known as a big multi view data set and all sequences are captured under 11 different conditions. Besides, three different variations are presented in this data set as shown in Figure 7 such as view angle, wearing a coat and carrying condition. For experimental results, 100 subjects' data is collected from all three variations and a strategy of 60:40 is made for training and testing. 10-fold validations are performed for all testing results. The recognition results are attained in two steps. First, using serial-based features fusion and without features selection algorithm and second, using a proposed threshold based parallel 
Table 3. Recognition results of CASIA-A data set using serial-based fusion and without features selection method

\begin{tabular}{|c|c|c|c|c|c|c|c|}
\hline \multirow[t]{2}{*}{ Classifiers } & \multicolumn{7}{|c|}{ Performance Measures } \\
\hline & TPR & FPR & PPV & FNR $(\%)$ & FDR & AUC & $\operatorname{CCR}(\%)$ \\
\hline DT & 0.934 & 0.324 & 93.46 & 5.3 & 6.53 & 0.960 & 94.7 \\
\hline LDA & 0.954 & 0.019 & 97.23 & 3.1 & 2.76 & 0.991 & 96.9 \\
\hline MSVM & 0.984 & 0.007 & 98.50 & 1.4 & 1.50 & 0.992 & 98.6 \\
\hline $\mathrm{KNN}$ & 0.981 & 0.008 & 98.20 & 1.6 & 1.80 & 0.992 & 98.4 \\
\hline EBT & 0.953 & 0.019 & 95.7 & 3.5 & 4.30 & 0.991 & 96.5 \\
\hline
\end{tabular}

Table 4. Confusion matrix of CASIA-A data set using serial-based fusion and without features selection method

\begin{tabular}{|l|l|l|l|}
\hline \multicolumn{1}{|c|}{ Class } & \multicolumn{1}{c|}{ Walk 45 View } & \multicolumn{1}{c|}{ Walk 90 View } & \multicolumn{1}{c|}{ Walk parallel } \\
\hline Walk 45 View & $\mathbf{9 6 . 4} \%$ & $2.7 \%$ & $1.0 \%$ \\
\hline Walk 90 View & $0.1 \%$ & $\mathbf{1 0 0 . 0} \%$ & $0.2 \%$ \\
\hline Walk parallel & $0.6 \%$ & $-\ldots$ & $\mathbf{9 9 . 4} \%$ \\
\hline
\end{tabular}

Table 5. Recognition results of proposed method for CASIA-A data set using threshold based parallel fusion and features selection method

\begin{tabular}{|l|l|l|l|l|l|l|l|l|l|l|}
\hline \multirow{2}{*}{ Classifiers } & \multicolumn{9}{|c|}{ Performance Measures } \\
\cline { 2 - 11 } & TPR & FPR & PPV & $\begin{array}{c}\text { FNR } \\
(\%)\end{array}$ & FDR & AUC & $\begin{array}{c}\text { TNR } \\
(\%)\end{array}$ & $\begin{array}{c}\text { NPV } \\
(\%)\end{array}$ & $\begin{array}{c}\text { FOR } \\
(\%)\end{array}$ & $\begin{array}{c}\text { CCR } \\
(\%)\end{array}$ \\
\hline DT & 0.938 & 0.032 & 94.03 & 5.0 & 5.96 & 0.963 & 94.29 & 95.74 & 4.26 & 95.0 \\
\hline LDA & 0.958 & 0.017 & 97.06 & 2.9 & 2.93 & 0.988 & 96.99 & 97.89 & 2.11 & 97.1 \\
\hline MSVM & $\mathbf{0 . 9 8 3}$ & $\mathbf{0 . 0 0 7}$ & $\mathbf{9 8 . 5 3}$ & $\mathbf{1 . 1}$ & $\mathbf{1 . 4 6}$ & 0.989 & $\mathbf{9 8 . 5 7}$ & $\mathbf{9 9 . 0 5}$ & $\mathbf{0 . 9 5}$ & $\mathbf{9 8 . 9}$ \\
\hline KNN & 0.973 & 0.025 & 98.36 & 1.4 & 1.63 & 0.992 & 97.04 & 97.19 & 2.81 & 98.6 \\
\hline EBT & 0.951 & 0.038 & 95.30 & 3.6 & 4.70 & $\mathbf{0 . 9 9 0}$ & 95.71 & 98.90 & 1.10 & 96.4 \\
\hline
\end{tabular}

Table 6. Confusion matrix of proposed method for CASIA-A data set

\begin{tabular}{|l|l|l|l|}
\hline \multicolumn{1}{|c|}{ Class } & \multicolumn{1}{c|}{ Walk 45 View } & \multicolumn{1}{c|}{ Walk 90 View } & \multicolumn{1}{c|}{ Walk parallel } \\
\hline Walk 45 View & $\mathbf{9 6 . 3 \%}$ & $2.9 \%$ & $0.9 \%$ \\
\hline Walk 90 View & $0.1 \%$ & $\mathbf{1 0 0 . 0 \%}$ & $0.2 \%$ \\
\hline Walk parallel & $0.6 \%$ & --- & $\mathbf{9 9 . 4 \%}$ \\
\hline
\end{tabular}

features fusion and features selection-based method. The recognition results of both steps are presented in Table 8 and Table 10. The MSVM results are also proved in Table 9 and Table 11. The proposed method achieved maximum recognition rate as $95.8 \%$ and recognition time is 3.234 FPS whereas step 1 recognition rate is $93.5 \%$ and execution time is 9.230 FPS which gives the authenticity of proposed system. Moreover, a comparison of presented method is conducted in Table 12 and Figure 8 which confirms the validity of proposed method. 
Table 7. Comparison of proposed method with existing techniques for CASIA-A data set

\begin{tabular}{|l|l|}
\hline \multicolumn{1}{|c|}{ Algorithms } & \multicolumn{1}{c|}{ CCR (\%) } \\
\hline Lee et al. (Lee, Hong et al., 2009) & 91.25 \\
\hline Nandini et al. (Nandini, Sindhu et al., 2011) & 78.75 \\
\hline Zeng et al. (Zeng, Wang et al., 2014) & 92.50 \\
\hline Proposed method & $\mathbf{9 8 . 9 0}$ \\
\hline
\end{tabular}

Table 8. Recognition results of CASIA-B data set using serial-based fusion and without features selection method

\begin{tabular}{|l|l|l|l|l|l|l|l|}
\hline \multirow{2}{*}{ Classifiers } & \multicolumn{7}{|c|}{ Performance Measures } \\
\cline { 2 - 8 } & \multicolumn{1}{|c|}{ TPR } & \multicolumn{1}{|c|}{ FPR } & \multicolumn{1}{c|}{ PPV } & FNR (\%) & \multicolumn{1}{c|}{ FDR } & AUC & CCR (\%) \\
\hline LDA & 0.851 & 0.074 & 85.43 & 14.8 & 14.56 & 0.962 & 85.2 \\
\hline MSVM & 0.933 & 0.032 & 93.56 & 06.5 & 06.43 & 0.975 & $\mathbf{9 3 . 5}$ \\
\hline KNN & 0.874 & 0.062 & 87.56 & 12.5 & 12.43 & 0.905 & 87.5 \\
\hline EBT & 0.865 & 0.067 & 86.83 & 13.4 & 13.16 & 0.949 & 86.6 \\
\hline
\end{tabular}

Table 9. Confusion matrix of CASIA-B data set using serial-based fusion and without features selection method

\begin{tabular}{|l|l|l|l|}
\hline \multicolumn{1}{|c|}{ Class } & \multicolumn{1}{c|}{ Walk 45 View } & \multicolumn{1}{c|}{ Walk 90 View } & \multicolumn{1}{c|}{ Walk parallel } \\
\hline Walk 45 View & $\mathbf{9 2 . 3} \%$ & $4.4 \%$ & $3.3 \%$ \\
\hline Walk 90 View & $1.6 \%$ & $\mathbf{9 4 . 3} \%$ & $4.2 \%$ \\
\hline Walk parallel & $2.1 \%$ & $4.1 \%$ & $\mathbf{9 3 . 8} \%$ \\
\hline
\end{tabular}

Table 10. Recognition results of proposed method for CASIA-B data set using threshold based parallel fusion and features selection method

\begin{tabular}{|l|l|l|l|l|l|l|l|l|l|l|}
\hline \multirow{2}{*}{ Classifier } & \multicolumn{10}{|c|}{ Performance Measures } \\
\cline { 2 - 12 } & TPR & FPR & $\mathbf{P P V}$ & $\begin{array}{c}\text { FNR } \\
(\boldsymbol{\%})\end{array}$ & FDR & AUC & $\begin{array}{c}\text { TNR } \\
(\%)\end{array}$ & $\begin{array}{c}\text { NPV } \\
(\%)\end{array}$ & $\begin{array}{c}\text { FOR } \\
(\%)\end{array}$ & $\begin{array}{c}\text { CCR } \\
(\%)\end{array}$ \\
\hline DT & 0.736 & 0.130 & 84.86 & 14.9 & 15.1 & 0.802 & 93.18 & 91.49 & 8.51 & 85.1 \\
\hline LDA & 0.946 & 0.026 & 94.60 & 5.4 & 5.4 & 0.992 & 96.29 & 97.90 & 2.1 & 94.6 \\
\hline MSVM & $\mathbf{0 . 9 5 6}$ & $\mathbf{0 . 0 2 0}$ & $\mathbf{9 6 . 0 0}$ & $\mathbf{4 . 2}$ & $\mathbf{4 . 0}$ & $\mathbf{0 . 9 9 4}$ & $\mathbf{9 5 . 4 1}$ & $\mathbf{9 7 . 1 1}$ & $\mathbf{2 . 8 9}$ & $\mathbf{9 5 . 8}$ \\
\hline KNN & 0.736 & 0.130 & 78.7 & 25.6 & 21.3 & 0.802 & 90.86 & 87.91 & 12.09 & 74.4 \\
\hline EBT & 0.888 & 0.053 & 90.06 & 10.7 & 9.93 & 0.975 & 94.70 & 92.26 & 7.74 & 89.3 \\
\hline
\end{tabular}

\section{CASIA-C Data Set}

This data set consists of 153 humans with four different walking conditions such as normal walk, slow walk, fast walk and walk with carrying item. This data set is generated by a low resolution thermal camera and all videos are captured in the night. 60:40 strategies are adopted with 10 fold validations. Two steps are conducted for recognition results. Proposed method with threshold based parallel fusion and features selection method achieved maximum recognition accuracy as $97.3 \%$ which is significantly 
Table 11. Confusion matrix of proposed method for CASIA-B data set

\begin{tabular}{|l|l|l|l|}
\hline \multicolumn{1}{|c|}{ Class } & \multicolumn{1}{|c|}{ Carrying Bag } & \multicolumn{1}{c|}{ Clothing } & \multicolumn{1}{c|}{ Normal Walk } \\
\hline Carrying Bag & $\mathbf{9 8 . 3 \%}$ & --- & $1.7 \%$ \\
\hline Clothing & $1.8 \%$ & $\mathbf{9 8 . 2 \%}$ & $0.2 \%$ \\
\hline Normal Walk & $5.8 \%$ & $3.8 \%$ & $\mathbf{9 0 . 4 \%}$ \\
\hline
\end{tabular}

Table 12. Comparison of proposed method with existing techniques for CASIA-B data set

\begin{tabular}{|l|l|}
\hline \multicolumn{1}{|c|}{ Algorithms } & \multicolumn{1}{c|}{ CCR (\%) } \\
\hline Liu et al. (Liu, Yin et al., 2011) & 97.67 \\
\hline Wang et al. (Wang, Zhang et al., 2012) & 91.57 \\
\hline Hu et al. (Hu, Wang et al., 2013) & 94.00 \\
\hline Lishani et al. (Lishani, Boubchir et al., 2014) & 83.00 \\
\hline Zeng et al. (Zeng, Wang et al., 2014) & 91.90 \\
\hline Rida et al. (Rida, Almaadeed et al., 2016) & 81.40 \\
\hline Rida et al. (Rida, Jiang et al., 2016) & 88.75 \\
\hline Lishani et al. (Lishani, Boubchir et al., 2016) & 90.14 \\
\hline Proposed method & $\mathbf{9 5 . 8 0}$ \\
\hline
\end{tabular}

Figure 8. Comparison of proposed method with existing techniques

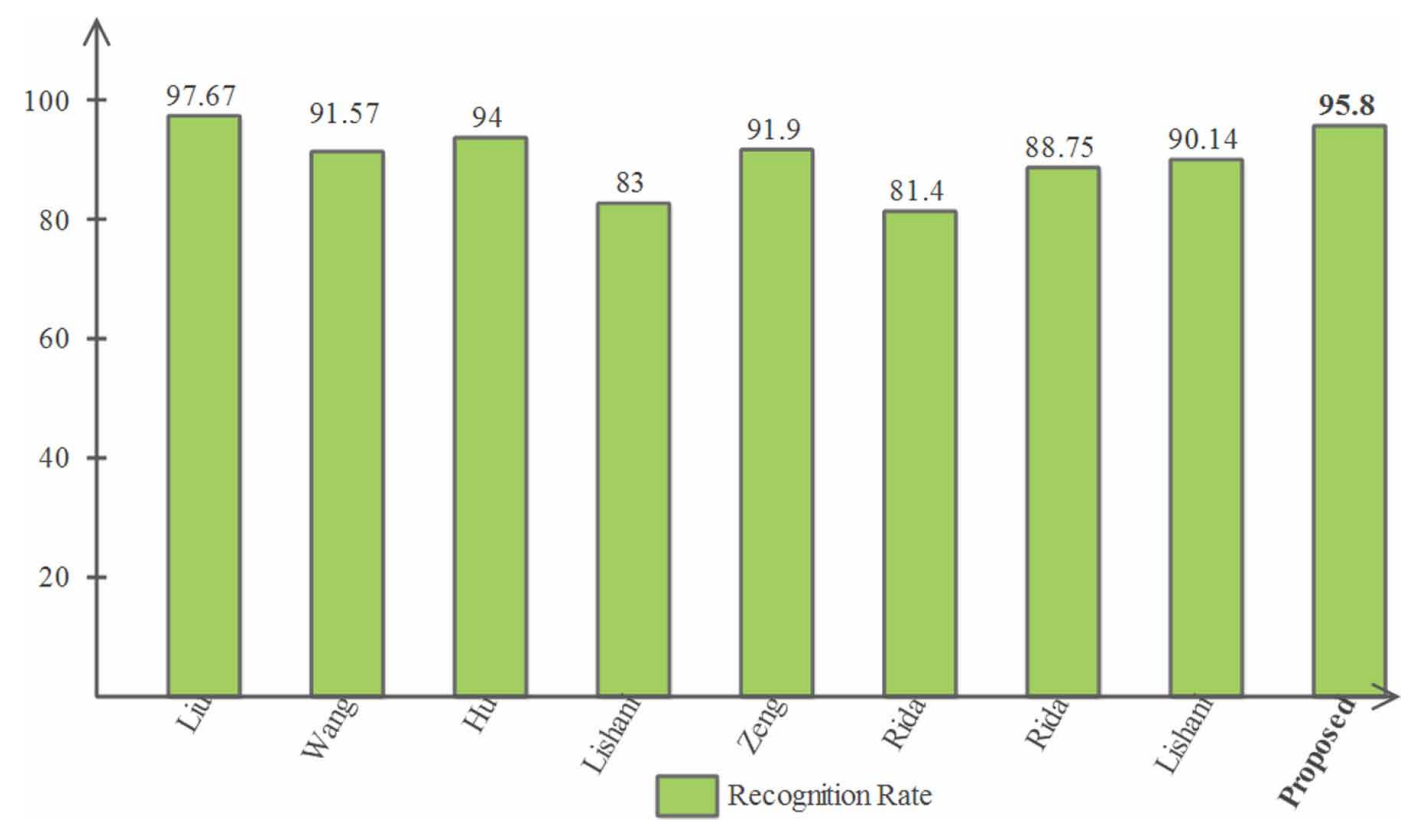


well as compared to step 1 recognition results of $96.9 \%$ with serial based features fusion and without features selection method. The recognition results of both steps are presented in Table 13 and Table 15 which are proved by Table 14 and Table 16. The execution time of proposed method for this data set is 2.009 FPS which is better than step 1 (5.967 FPS) as shown in Figure 9. Moreover, in Table 17 , a comparison is conducted which shows realism of presented method.

\section{DISCUSSION}

The proposed method is interpreted in this section with visual and tabular results. As shown in Figure 1, four steps are performed in this method. The preprocessing step removes the noisy effects and clears the foreground as shown in Figures 2, 3, and 4. After that, multiple features are fused using threshold based parallel fusion and best features are selected by the Euclidian distance-based method as presented in Figure 5. Then the selected features are fed to MSVM for gait recognition. The visual effects of proposed method are shown in Figure 6. For experimental results, the proposed method is compared with serial-based features fusion and without features selection methods on all

Table 13. Recognition results of CASIA-C data set using serial based fusion and without features selection method

\begin{tabular}{|l|l|l|l|l|l|l|l|}
\hline \multirow{2}{*}{ Classifier } & \multicolumn{7}{|c|}{ Performance Measures } \\
\cline { 2 - 8 } & \multicolumn{1}{|c|}{ TPR } & \multicolumn{1}{|c|}{ FPR } & \multicolumn{1}{|c|}{ PPV } & \multicolumn{1}{|c|}{ FNR $(\%)$} & \multicolumn{1}{c|}{ FDR } & AUC & CCR (\%) \\
\hline LDA & 0.868 & 0.041 & 89.47 & 11.8 & 10.52 & 0.976 & 88.2 \\
\hline MSVM & 0.964 & 0.010 & 97.10 & 3.1 & 2.80 & 0.995 & $\mathbf{9 6 . 9}$ \\
\hline KNN & 0.965 & 0.010 & 97.25 & 3.1 & 2.75 & 0.977 & $\mathbf{9 6 . 9}$ \\
\hline EBT & 0.958 & 0.011 & 96.70 & 3.5 & 3.30 & 0.993 & 96.5 \\
\hline
\end{tabular}

Table 14. Confusion matrix of CASIA-C data set using serial based fusion and without features selection method

\begin{tabular}{|l|l|l|l|l|}
\hline \multicolumn{1}{|c|}{ Class } & \multicolumn{1}{|c|}{ Carrying Bag } & \multicolumn{1}{c|}{ Fast Walk } & \multicolumn{1}{c|}{ Normal Walk } & \multicolumn{1}{c|}{ Slow Walk } \\
\hline Carrying Bag & $\mathbf{9 9 . 4 \%}$ & $0.3 \%$ & --- & $0.3 \%$ \\
\hline Fast Walk & --- & $\mathbf{9 1 . 8} \%$ & $0.4 \%$ & $7.8 \%$ \\
\hline Normal Walk & --- & $1.6 \%$ & $\mathbf{9 6 . 6 \%}$ & $1.9 \%$ \\
\hline Slow Walk & --- & $1.4 \%$ & $0.2 \%$ & $\mathbf{9 8 . 4 \%}$ \\
\hline
\end{tabular}

Table 15. Recognition results of proposed method for CASIA-C data set using threshold based parallel fusion and features selection method

\begin{tabular}{|l|l|l|l|l|l|l|l|l|l|l|l|}
\hline \multirow{2}{*}{ Classifiers } & \multicolumn{9}{|c|}{ Performance Measures } \\
\cline { 2 - 12 } & TPR & FPR & PPV & $\begin{array}{c}\text { FNR } \\
(\%)\end{array}$ & FDR & AUC & $\begin{array}{c}\text { TNR } \\
(\%)\end{array}$ & $\begin{array}{c}\text { NPV } \\
(\%)\end{array}$ & $\begin{array}{c}\text { FOR } \\
(\%)\end{array}$ & $\begin{array}{c}\text { CCR } \\
(\%)\end{array}$ \\
\hline LDA & 0.869 & 0.040 & 89.60 & 11.8 & 10.4 & 0.975 & 91.10 & 89.98 & 10.02 & 88.2 \\
\hline KNN & 0.966 & 0.009 & 97.27 & 3.0 & 2.72 & $\mathbf{0 . 9 9 5}$ & 98.52 & 97.75 & 2.25 & 97.0 \\
\hline MSVM & $\mathbf{0 . 9 6 9}$ & $\mathbf{0 . 0 0 8}$ & $\mathbf{9 7 . 4 5}$ & $\mathbf{2 . 7}$ & $\mathbf{2 . 5 5}$ & 0.979 & $\mathbf{9 7 . 4 5}$ & $\mathbf{9 8 . 8 0}$ & $\mathbf{1 . 2}$ & $\mathbf{9 7 . 3}$ \\
\hline EBT & 0.957 & 0.012 & 96.82 & 3.6 & 3.17 & 0.987 & 97.78 & 97.41 & 2.59 & 96.4 \\
\hline
\end{tabular}


Table 16. Confusion matrix of proposed method for CASIA-C data set

\begin{tabular}{|l|l|l|l|l|}
\hline \multicolumn{1}{|c|}{ Class } & \multicolumn{1}{c|}{ Carrying Bag } & \multicolumn{1}{c|}{ Fast Walk } & \multicolumn{1}{c|}{ Normal Walk } & \multicolumn{1}{c|}{ Slow Walk } \\
\hline Carrying Bag & $\mathbf{9 9 . 1 \%}$ & $0.8 \%$ & $0.2 \%$ & $0.3 \%$ \\
\hline Fast Walk & $0.3 \%$ & $\mathbf{9 6 . 8} \%$ & $1.0 \%$ & $2.9 \%$ \\
\hline Normal Walk & $0.6 \%$ & $1.2 \%$ & $\mathbf{9 8 . 1 \%}$ & $1.1 \%$ \\
\hline Slow Walk & --- & $1.2 \%$ & $0.6 \%$ & $\mathbf{9 5 . 8 \%}$ \\
\hline
\end{tabular}

Table 17. Comparison of proposed method with existing techniques for CASIA-C data set

\begin{tabular}{|l|l|}
\hline \multicolumn{1}{|c|}{ Algorithms } & \multicolumn{1}{|c|}{ CCR (\%) } \\
\hline Tan et al. (Tan, Huang et al., 2006) & 82.25 \\
\hline Zhang et al. (Zhang, Zhao et al., 2010) & 87.01 \\
\hline Zeng et al. (Zeng, Wang et al., 2014) & 90.20 \\
\hline Proposed method & $\mathbf{9 7 . 3 0}$ \\
\hline
\end{tabular}

Figure 9. Execution time of selected datasets

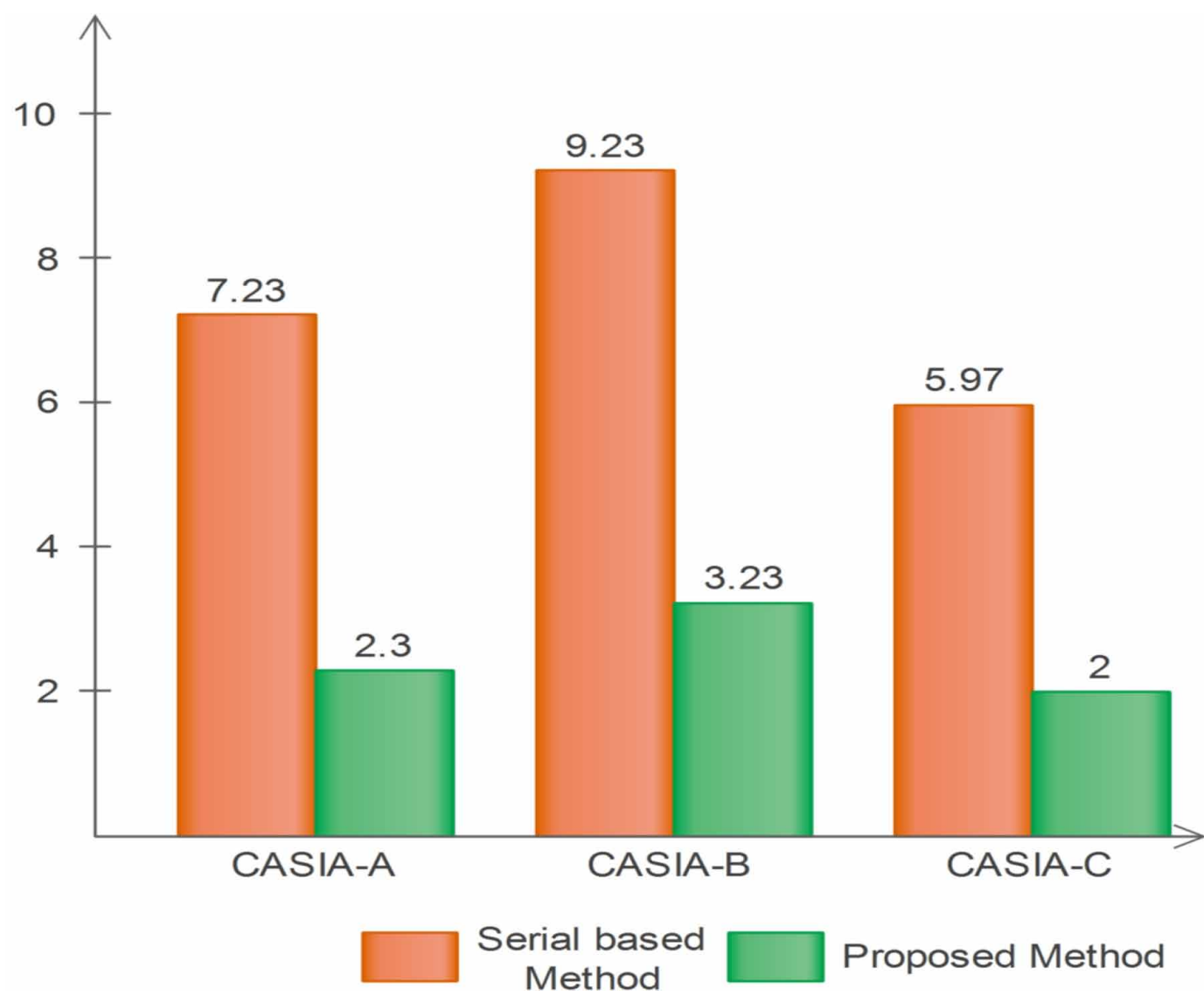


three selected data sets and their results are presented in Table 3, Table 5, Table 8, Table 10, Table 13 and Table 15, respectively. Table 3 and Table 5 represent the recognition accuracy on CASIA-A data set as $98.6 \%$ without features selection and $98.9 \%$ with features selection. In Table 8 and Table 10 , the CASIA-B data set results are given and achieved accuracy without features selection and with features selection as $93.5 \%$ and $95.8 \%$, respectively. Similarly, in Table 13 and Table 15, results are achieved on CASIA-C data set and achieved recognition rate as $96.9 \%$ and $97.3 \%$. The results show that the proposed fusion and selection method is outperformed on selected data sets. The recognition results are also proved by Tables 4, 6, 9, 11, 14 and 16, respectively.

Additionally, few new performance matrices are calculated for proposed parallel fusion and selection approach such as TNR, Negative Predictive Value (NPV), False Omission Rate (FOR) and FPR as given in Tables 5, 10, and 15. In Table 5, these calculated measures for CASIA-A data set on MSVM are $98.57 \%, 99.05 \%, 0.95 \%$ and 0.007 , respectively. Similarly in Table 10 and Table 15, these measures are calculated and values on MSVM are $95.41 \%, 97.11 \%, 2.89 \%$, and 0.020 for CASIA B dataset and $97.45 \%, 98.80 \%, 1.2 \%$, and 0.008 for CASIA C dataset. These measures show that the proposed method accurately recognized human gait in the video sequences.

The classification comparison on all selected data sets is shown in Figure 10 which proves that MSVM performs significantly well. Moreover, the execution time of proposed method is considerably well as compared to a serial-based method and without features selection as shown in Figure 9. Finally, the proposed method is compared with existing methods as presented in Tables 7, 12 and 17 which shows its authenticity.

\section{CONCLUSION AND FUTURE WORK}

In this article, a method for automatic HGR is presented in the given video sequences using parallel features fusion and Euclidian distance based best features selection. Four series of steps are performed like preprocessing, human extraction, multiple features extraction and best features selection and recognition. Initially, the raw video frames are enhanced and later on optical flow and background subtraction method is utilized for human extraction. Texture, HOG and geometric features are fused by using a threshold based parallel method and best features are chosen by determining the Euclidian distance between them. The selected features are fed to MSVM for final recognition. Three publicly available data sets such as CASIA-A, CASIA-B and CASIA-C are utilized and achieved recognition rates as $98.90 \%, 95.80 \%$ and $97.30 \%$, respectively. The proposed system is also compared with existing methods which shows the effectiveness of presented method.

However, this work has few limitations such as camera view point and dynamic background. This method degrades the recognition accuracy when several cameras are utilized for gait monitoring. Moreover, the dynamic environment is not compatible for this approach because it is proposed for static environment. In future, the above system can be converted into deep learning and deep features can be extracted instead of shape, texture and geometric. The deep features efficiently work in real time video sequences and also take less time for system execution. In addition, through deep learning, challenges of dynamic environment can be solved. Moreover, the deep features model can be applied on big data sets to test the scalability and execution of the system. 
Figure 10. Comparison of MSVM recognition accuracy with LDA, DT, and KNN on all three selected datasets

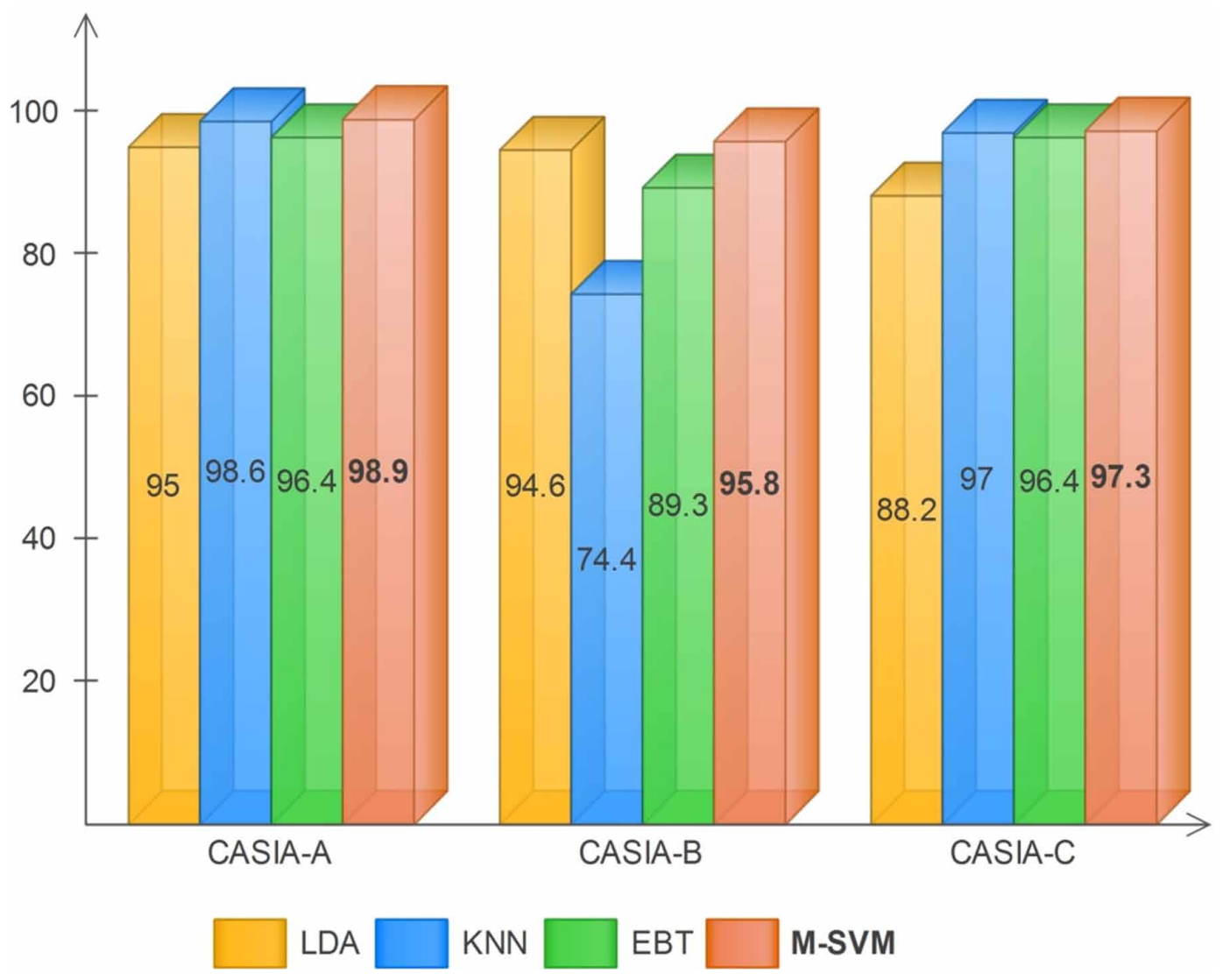




\section{REFERENCES}

Abdullah, B. A., \& El-Alfy, E.-S. M. (2015). Statistical Gabor-Based Gait Recognition Using Region-Level Analysis. In Proceedings of the 2015 IEEE European Modelling Symposium (EMS). IEEE. doi:10.1109/ EMS.2015.30

Al-Tayyan, A., Assaleh, K., \& Shanableh, T. (2017). Decision-level fusion for single-view gait recognition with various carrying and clothing conditions. Image and Vision Computing, 61, 54-69. doi:10.1016/j. imavis.2017.02.004

Alotaibi, M., \& Mahmood, A. (2015). Improved gait recognition based on specialized deep convolutional neural networks. In Proceedings of the 2015 IEEE Applied Imagery Pattern Recognition Workshop (AIPR). IEEE. doi:10.1109/AIPR.2015.7444550

Amin, J., Sharif, M., Yasmin, M., Ali, H., \& Fernandes, S. L. (2017). A method for the detection and classification of diabetic retinopathy using structural predictors of bright lesions. Journal of Computational Science, 19, 153-164. doi:10.1016/j.jocs.2017.01.002

Amin, J., Sharif, M., Yasmin, M., \& Fernandes, S. L. (2017). A distinctive approach in brain tumor detection and classification using MRI. Pattern Recognition Letters. doi:10.1016/j.patrec.2017.10.036

Amin, J., Sharif, M., Yasmin, M., \& Fernandes, S. L. (2018). Big data analysis for brain tumor detection: Deep convolutional neural networks. Future Generation Computer Systems, 87, 290-297. doi:10.1016/j. future.2018.04.065

Ansari, G. J., Shah, J. H., Yasmin, M., Sharif, M., \& Fernandes, S. L. (2018). A novel machine learning approach for scene text extraction. Future Generation Computer Systems, 87, 328-340. doi:10.1016/j.future.2018.04.074

Aqmar, M. R., Fujihara, Y., Makihara, Y., \& Yagi, Y. (2014). Gait recognition by fluctuations. Computer Vision and Image Understanding, 126, 38-52. doi:10.1016/j.cviu.2014.05.004

Arora, P., Hanmandlu, M., \& Srivastava, S. (2015). Gait based authentication using gait information image features. Pattern Recognition Letters, 68, 336-342. doi:10.1016/j.patrec.2015.05.016

Barron, J. L., Fleet, D. J., \& Beauchemin, S. S. (1994). Performance of optical flow techniques. International Journal of Computer Vision, 12(1), 43-77.

Bhushan, S. B., Danti, A., \& Fernandes, S. L. (2018). Integer Representation and B-Tree for Classification of Text Documents: An Integrated Approach. In Information and Decision Sciences (pp. 481-490). Springer Singapore.

Bokhari, F., Syedia, T., Sharif, M., Yasmin, M., \& Fernandes, S. L. (2018). Fundus image segmentation and feature extraction for the detection of glaucoma: A new approach. Current Medical Imaging Reviews, 14(1), 77-87. doi:10.2174/1573405613666170405145913

Choudhury, S. D., \& Tjahjadi, T. (2015). Robust view-invariant multiscale gait recognition. Pattern Recognition, 48(3), 798-811. doi:10.1016/j.patcog.2014.09.022

Deng, M., Wang, C., Cheng, F., \& Zeng, W. (2017). Fusion of spatial-temporal and kinematic features for gait recognition with deterministic learning. Pattern Recognition, 67, 186-200. doi:10.1016/j.patcog.2017.02.014

Dey, N., Ashour, A. S., \& Hassanien, A. E. (2018). Feature detectors and descriptors generations with numerous images and video applications: a recap. In Computer Vision: Concepts, Methodologies, Tools, and Applications (pp. 571-600). Hershey, PA: IGI Global. doi:10.4018/978-1-5225-5204-8.ch022

Fernandes, S. L., \& Bala, G. J. (2016). Fusion of sparse representation and dictionary matching for identification of humans in uncontrolled environment. Computers in Biology and Medicine, 76, 215-237. doi:10.1016/j. compbiomed.2016.07.007 PMID:27498411

Fernandes, S. L., Gurupur, V. P., Lin, H., \& Martis, R. J. (2017). A Novel fusion approach for early lung cancer detection using computer aided diagnosis techniques. Journal of Medical Imaging and Health Informatics, 7(8), 1841-1850. doi:10.1166/jmihi.2017.2280

Gupta, J. P., Dixit, P., \& Semwal, V. B. (2014). Analysis of Gait Pattern to Recognize the Human Activities. IJIMAI, 2(7), 7-16. doi:10.9781/ijimai.2014.271 
Hu, M., Wang, Y., Zhang, Z., Zhang, D., \& Little, J. J. (2013). Incremental learning for video-based gait recognition with LBP flow. IEEE Transactions on Cybernetics, 43(1), 77-89. doi:10.1109/TSMCB.2012.2199310 PMID:22692925

Islam, M. S., Islam, M. R., Hossain, M. A., Ferworn, A., \& Molla, M. K. I. (2017). Subband entropy-based features for clothing invariant human gait recognition. Advanced Robotics, 31(10), 519-530. doi:10.1080/016 91864.2017.1283249

Khan, M. A., Akram, T., Sharif, M., Javed, M. Y., Muhammad, N., \& Yasmin, M. (2018). An implementation of optimized framework for action classification using multilayers neural network on selected fused features. Pattern Analysis \& Applications, 1-21.

Khan, M. A., Akram, T., Sharif, M., Shahzad, A., Aurangzeb, K., Alhussein, M., \& Altamrah, A. et al. (2018). An implementation of normal distribution based segmentation and entropy controlled features selection for skin lesion detection and classification. BMC Cancer, 18(1), 638. doi:10.1186/s12885-018-4465-8 PMID:29871593

Khan, M. A., Sharif, M., Javed, M. Y., Akram, T., Yasmin, M., \& Saba, T. (2017). License number plate recognition system using entropy-based features selection approach with SVM. IET Image Processing, 12(2), 200-209. doi:10.1049/iet-ipr.2017.0368

Khan, M. W., Sharif, M., Yasmin, M., \& Fernandes, S. L. (2016). A new approach of cup to disk ratio based glaucoma detection using fundus images. Journal of Integrated Design \& Process Science, 20(1), 77-94. doi:10.3233/jid-2016-0004

Lee, H., Hong, S., \& Kim, E. (2009). Neural network ensemble with probabilistic fusion and its application to gait recognition. Neurocomputing, 72(7), 1557-1564. doi:10.1016/j.neucom.2008.09.009

Liaqat, A., Khan, M. A., Shah, J. H., Sharif, M., Yasmin And, M., \& Fernandes, S. L. (2018). Automated Ulcer And Bleeding Classification From Wce Images Using Multiple Features Fusion And Selection. Journal of Mechanics in Medicine and Biology, 18(04), 1850038. doi:10.1142/S0219519418500380

Lishani, A. O., Boubchir, L., \& Bouridane, A. (2014). Haralick features for GEI-based human gait recognition. In Proceedings of the 2014 26th International Conference on Microelectronics (ICM). IEEE.

Lishani, A. O., Boubchir, L., Khalifa, E., \& Bouridane, A. (2016). Gabor filter bank-based GEI features for human Gait recognition. In Proceedings of the 2016 39th International Conference on Telecommunications and Signal Processing (TSP). IEEE. doi:10.1109/TSP.2016.7760962

Lishani, A. O., Boubchir, L., Khalifa, E., \& Bouridane, A. (2017). Gait recognition based on wavelet features with spectral regression kernel discriminant analysis. In Proceedings of the 2017 40th International Conference on, Telecommunications and Signal Processing (TSP). IEEE. doi:10.1109/TSP.2017.8076096

Lishani, A. O., Boubchir, L., Khalifa, E., \& Bouridane, A. (2017). Human gait recognition based on Haralick features. Signal, Image and Video Processing, 11(6), 1123-1130.

Liu, L., Yin, Y., Qin, W., \& Li, Y. (2011). Gait recognition based on outermost contour. International Journal of Computational Intelligence Systems, 4(5), 1090-1099. doi:10.1080/18756891.2011.9727857

Moustakas, K., Tzovaras, D., \& Stavropoulos, G. (2010). Gait recognition using geometric features and soft biometrics. IEEE Signal Processing Letters, 17(4), 367-370. doi:10.1109/LSP.2010.2040927

Nandini, C., Sindhu, K., \& Kumar, C. R. (2011). Gait recognition by combining wavelets and geometrical features. In Proceedings of the 2011 2nd International Conference on, Intelligent Agent and Multi-Agent Systems (IAMA). IEEE. doi:10.1109/IAMA.2011.6049003

Naqi, S., Sharif, M., Yasmin, M., \& Fernandes, S. L. (2018). Lung nodule detection using polygon approximation and hybrid features from CT images. Current Medical Imaging Reviews, 14(1), 108-117. doi:10.2174/157340 5613666170306114320

Nasir, M., Attique Khan, M., Sharif, M., Lali, I. U., Saba, T., \& Iqbal, T. (2018). An improved strategy for skin lesion detection and classification using uniform segmentation and feature selection based approach. Microscopy Research and Technique, 81(6), 528-543. doi:10.1002/jemt.23009 PMID:29464868

Nida, N., Sharif, M., Khan, M. U. G., Yasmin, M., \& Fernandes, S. L. (2016). A framework for automatic colorization of medical imaging. The IIOAB Journal, 7, 202-209. 
Piccardi, M. (2004). Background subtraction techniques: a review. In Proceedings of the 2004 IEEE international conference on Systems, man and cybernetics. IEEE.

Premalatha, G., Williams, A. T., \& Abraham, S. K. (2016). View-invariant gait recognition using gait energy image (GEI). Biometrics and Bioinformatics, 8(6), 161-167.

Raja, N. S. M., Fernandes, S., Dey, N., Satapathy, S. C., \& Rajinikanth, V. (2018). Contrast enhanced medical MRI evaluation using Tsallis entropy and region growing segmentation. Journal of Ambient Intelligence and Humanized Computing, 1-12.

Rajinikanth, V., Fernandes, S. L., Bhushan, B., \& Sunder, N. R. (2018). Segmentation and analysis of brain tumor using Tsallis entropy and regularised level set. In Proceedings of 2 nd International Conference on MicroElectronics, Electromagnetics and Telecommunications. Springer. doi:10.1007/978-981-10-4280-5_33

Rajinikanth, V., Madhavaraja, N., Satapathy, S. C., \& Fernandes, S. L. (2017). Otsu's Multi-Thresholding and Active Contour Snake Model to Segment Dermoscopy Images. Journal of Medical Imaging and Health Informatics, 7(8), 1837-1840. doi:10.1166/jmihi.2017.2265

Rajinikanth, V., Satapathy, S. C., Fernandes, S. L., \& Nachiappan, S. (2017). Entropy based segmentation of tumor from brain MR images-a study with teaching learning based optimization. Pattern Recognition Letters, 94, 87-95. doi:10.1016/j.patrec.2017.05.028

Ranjan, R., Arya, R., Fernandes, S. L., Sravya, E., \& Jain, V. (2018). A Fuzzy Neural Network Approach for Automatic K-Complex Detection in Sleep EEG Signal. Pattern Recognition Letters, 115, 74-83. doi:10.1016/j. patrec.2018.01.001

Raza, M., Sharif, M., Yasmin, M., Khan, M. A., Saba, T., \& Fernandes, S. L. (2018). Appearance based pedestrians' gender recognition by employing stacked auto encoders in deep learning. Future Generation Computer Systems, 88, 28-39. doi:10.1016/j.future.2018.05.002

Rida, I., Almaadeed, S., \& Bouridane, A. (2016). Gait recognition based on modified phase-only correlation. Signal, Image and Video Processing, 10(3), 463-470. doi:10.1007/s11760-015-0766-4

Rida, I., Jiang, X., \& Marcialis, G. L. (2016). Human body part selection by group lasso of motion for model-free gait recognition. IEEE Signal Processing Letters, 23(1), 154-158. doi:10.1109/LSP.2015.2507200

Ryu, J., \& Kamata, S.-i. (2011). Front view gait recognition using spherical space model with human point clouds. In Proceedings of the 2011 18th IEEE International Conference on Image Processing (ICIP). IEEE. doi:10.1109/ICIP.2011.6116351

Saeys, Y., I. Inza and P. Larrañaga (2007). A review of feature selection techniques in bioinformatics. Bioinformatics, 23(19), 2507-2517.

Shah, J. H., Chen, Z., Sharif, M., Yasmin, M., \& Fernandes, S. L. (2017). A novel biomechanics-based approach for person re-identification by generating dense color sift salience features. Journal of Mechanics in Medicine and Biology, 17(7), 1740011. doi:10.1142/S0219519417400115

Shah, J. H., Sharif, M., Yasmin, M., \& Fernandes, S. L. (2017). Facial expressions classification and false label reduction using LDA and threefold SVM. Pattern Recognition Letters. doi:10.1016/j.patrec.2017.06.021

Sharif, M., Khan, M. A., Akram, T., Javed, M. Y., Saba, T., \& Rehman, A. (2017). A framework of human detection and action recognition based on uniform segmentation and combination of Euclidean distance and joint entropy-based features selection. EURASIP Journal on Image and Video Processing, 2017(1), 89. doi:10.1186/ s13640-017-0236-8

Sharif, M., Khan, M. A., Faisal, M., Yasmin, M., \& Fernandes, S. L. (2018). A framework for offline signature verification system: Best features selection approach. Pattern Recognition Letters. doi:10.1016/j. patrec.2018.01.021

Sharif, M., Khan, M. A., Iqbal, Z., Azam, M. F., Lali, M. I. U., \& Javed, M. Y. (2018). Detection and classification of citrus diseases in agriculture based on optimized weighted segmentation and feature selection. Computers and Electronics in Agriculture, 150, 220-234. doi:10.1016/j.compag.2018.04.023

Tan, D., Huang, K., Yu, S., \& Tan, T. (2006). Efficient night gait recognition based on template matching. In Proceedings of the 18th International Conference on Pattern Recognition ICPR 2006. IEEE. 
Wang, C., Li, Z., Dey, N., Li, Z., Ashour, A. S., Fong, S. J., \& Shi, F. et al. (2018). Histogram of oriented gradient based plantar pressure image feature extraction and classification employing fuzzy support vector machine. Journal of Medical Imaging and Health Informatics, 8(4), 842-854. doi:10.1166/jmihi.2018.2310

Wang, C., Zhang, J., Wang, L., Pu, J., \& Yuan, X. (2012). Human identification using temporal information preserving gait template. IEEE Transactions on Pattern Analysis and Machine Intelligence, 34(11), 2164-2176. doi:10.1109/TPAMI.2011.260 PMID:22201053

Wang, J., Garcia, E. B., Yu, S., \& Zhang, D. (2017). Windowed DMD for Gait Recognition Under Clothing and Carrying Condition Variations. In Proceedings of the Chinese Conference on Biometric Recognition. Springer. doi:10.1007/978-3-319-69923-3_52

Wang, L., Tan, T., Ning, H., \& Hu, W. (2003). Silhouette analysis-based gait recognition for human identification. IEEE Transactions on Pattern Analysis and Machine Intelligence, 25(12), 1505-1518. doi:10.1109/ TPAMI.2003.1251144

Wu, Z., Huang, Y., Wang, L., Wang, X., \& Tan, T. (2017). A comprehensive study on cross-view gait based human identification with deep cnns. IEEE Transactions on Pattern Analysis and Machine Intelligence, 39(2), 209-226. doi:10.1109/TPAMI.2016.2545669 PMID:27019478

Yasmin, M., Sharif, M., Irum, I., Mehmood, W., \& Fernandes, S. L. (2016). Combining multiple color and shape features for image retrieval. The IIOAB Journal, 7(32), 97-110.

Yu, S., Chen, H., Wang, Q., Shen, L., \& Huang, Y. (2017). Invariant feature extraction for gait recognition using only one uniform model. Neurocomputing, 239, 81-93. doi:10.1016/j.neucom.2017.02.006

Zeng, W., \& Wang, C. (2013). Silhouette-based gait recognition via deterministic learning. In Proceedings of the International Conference on Brain Inspired Cognitive Systems. Springer. doi:10.1007/978-3-642-38786-9_1

Zeng, W., \& Wang, C. (2016). View-invariant gait recognition via deterministic learning. Neurocomputing, 175, 324-335. doi:10.1016/j.neucom.2015.10.065

Zeng, W., Wang, C., \& Li, Y. (2014). Model-based human gait recognition via deterministic learning. Cognitive Computation, 6(2), 218-229. doi:10.1007/s12559-013-9221-4

Zeng, W., Wang, C., \& Yang, F. (2014). Silhouette-based gait recognition via deterministic learning. Pattern Recognition, 47(11), 3568-3584. doi:10.1016/j.patcog.2014.04.014

Zhang, E., Zhao, Y., \& Xiong, W. (2010). Active energy image plus 2DLPP for gait recognition. Signal Processing, 90(7), 2295-2302. doi:10.1016/j.sigpro.2010.01.024

Zhao, G., \& Pietikainen, M. (2007). Dynamic texture recognition using local binary patterns with an application to facial expressions. IEEE Transactions on Pattern Analysis and Machine Intelligence, 29(6), 915-928. doi:10.1109/ TPAMI.2007.1110 PMID:17431293 
Muhammad Sharif, PhD (IEEE Senior Member) is Associate Professor at COMSATS University Islamabad, Wah Campus Pakistan. He completed his PhD in research area of Image Processing from COMSATS Institute of IT, Islamabad Pakistan in 2013 and MS (CS) from the same institute in 2007. He earned his Master's in Computer Science from Quaid-e-Azam University, Islamabad Pakistan in 1995. Around 150 research publications in IF, SCI and ISI journals and national and international conferences are on his credit. So far 35 MS (CS) theses are supervised by him and currently supervising $5 \mathrm{PhD}$ (CS) students. Other $10 \mathrm{PhD}(\mathrm{CS})$ students are under his co-supervision. More than 300 undergraduate students have successfully completed their degree project with him. He was also evaluator of $3 \mathrm{PhD}$ (CS) and $30 \mathrm{MS}$ (CS) theses. His research interests are medical imaging, biometrics, computer vision, machine learning, and agriculture plants. COMSATS Research Productivity Award is continuously being given to him from 2011 till now. He served in TPC for IEEE FIT 2014-17 and currently serving as Associate Editor for IEEE Access, Guest Editor of Special Issues and reviewer for well reputed journals. He is into teaching field from 1996 till now with four years (2008-2011) as Head of Department of Computer Science, COMSATS Institute of IT, Wah Campus Pakistan achieved the targeted outputs.

Muhammad Attique completed BS and MS degrees in Computer Science from COMSATS Institute of Information Technology Wah. Currently, he is Lecturer at HITEC University. The research interest is including in pattern recognition, and computer vision (human gait recognition, human action recognition, biometric, and algorithms).

Mussarat Yasmin, PhD is Assistant Professor at COMSATS, Wah Cantt Pakistan. Her area of specialization is Image Processing. She is in education field since 1993. She has so far 30 research publications in IF, SCI and ISI journals as well as in national and international conferences. A number of undergraduate projects are complete under her supervision. She is currently supervising $5 \mathrm{PhD}$ (CS) students. She is gold medallist in MS (CS) from IQRA University, Pakistan. She is getting COMSATS research productivity award since 2012. Her research interests include Neural Network, Algorithms design and Analysis, Machine Learning and Image processing.

Tanzila Saba earned PhD in document information security and management from Faculty of Computing Universiti Teknologi Malaysia (UTM), Malaysia in 2012. She won best student award in the Faculty of Computing UTM for 2012. Currently, she is serving as Associate Prof. in College of Computer and Information Sciences Prince Sultan University Riyadh KSA. Her primary research focus in the recent years is Bioinformatics and Applied Soft Computing. She has above two hundred publications that have around 4000 citations with h-index 39 . She received best researcher awards in PSU for 2014, 2015,2016. Due to her excellent research achievement, she is included in Marquis Who's Who (S \& T) 2012." Currently she is editor of several reputed journals and on panel of TPC of international conferences and IEEE senior member.

Urcun John Tanik is an assistant professor in the Texas A\&M system with interests in the convergence of cyberphysical systems/IOT data science and engineering applications with STEM educational objectives. 